\title{
Dye-doped silica nanoparticles: synthesis, surface chemistry and bioapplications
}

\author{
Vladimir Gubala ${ }^{1}$, Giorgia Giovannini ${ }^{2}$, Filip Kunc ${ }^{3}$, Marco P. Monopoli ${ }^{4}$ and Colin J. Moore ${ }^{5 *}$ (D)
}

\section{${ }^{*}$ Correspondence:}

colin.moore@iit.it

${ }^{5}$ Istituto Italiano Tecnologia,

30 Via Morego, 16163 Genoa, Italy

Full list of author information is available at the end of the article

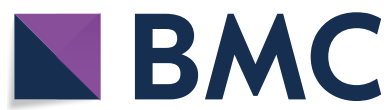

(C) The Author(s) 2020. This article is licensed under a Creative Commons Attribution 4.0 International License, which permits use, sharing, adaptation, distribution and reproduction in any medium or format, as long as you give appropriate credit to the original author(s) and the source, provide a link to the Creative Commons licence, and indicate if changes were made. The images or other third party material in this article are included in the article's Creative Commons licence, unless indicated otherwise in a credit line to the material. If material is not included in the article's Creative Commons licence and your intended use is not permitted by statutory regulation or exceeds the permitted use, you will need to obtain permission directly from the copyright holder. To view a copy of this licence, visit http://creativecommons.org/ licenses/by/4.0/. The Creative Commons Public Domain Dedication waiver (http://creativecommons.org/publicdomain/zero/1.0/) applies

to the data made available in this article, unless otherwise stated in a credit line to the data.
Keywords: Microporous, Silica nanoparticle, Dye loading, Cancer, Toxicity, Protein corona, Immunoassay, Diagnostics, Bioimaging, Drug delivery \begin{abstract}
understood, flexible chemistry and apparent biocompatibility. The ability to couple various siloxane precursors with fluorescent dyes and to be subsequently incorporated nanomaterials to specific optical requirements in biological experimentation. Consequently, this class of nanomaterial has been used in applications across immunodiagnostics, drug delivery and human-trial bioimaging in cancer research. thesis methor gies employed to generate biomolecule-coated nanoparticles. The use of dye-doped provided and possible future directions in the field are highlighted. Other non-cancerrelated applications involving silica nanoparticles are also briefly discussed. Importantly, the impact of how the protein corona has changed our understanding of NP interacbe favourably manipulated.
\end{abstract}

Conclusions: Dye-doped silica nanoparticles have found success in the immunodiagthe case for the vast majority of nanomaterials intended for cancer therapy. This is hampered by the need for more human-like disease models and the lack of standardisation the protein corona have improved the understanding of fundamental bio-nano interactions, and will undoubtedly assist in the translation of silica nanoparticles for disease treatment to the clinic. 


\section{Background}

As technological capacities continue to progress, scientists have made an increasing number of discoveries of molecular mechanisms underlying biological processes. This is largely due to the identification of specific intracellular events and protein-receptor interactions, e.g. antigen-antibody recognition. Endeavours in biotechnology are now therefore focussed on the detection and monitoring of particular chemical interactions of small targets that occur at low concentrations, such as individual cells (Maltez-da Costa et al. 2012), nucleic acids (Xiao et al. 2007), proteins (Giovannini and De Angelis 2019) and small molecules (Porchetta et al. 2012). The requirement to detect such entities has ultimately led to the development of similarly sized probes and, consequently, modern biological applications are now highly multidisciplinary and encompass a range of physiochemical fields like colloidal science, materials science and supramolecular chemistry for detecting, monitoring, and treating diseases.

A variety of new materials have been developed for nano-biotechnological purposes and nanoparticles (NP) are by far the most versatile and heavily studied class of nanomaterial. Numerous terms for 'nanoparticle' have been defined by various national and international regulatory bodies and typically require a material to have at least one dimension to be between 1 and $100 \mathrm{~nm}$ (Miernicki et al. 2019). However, despite these definitions, many researchers regularly use the term 'nano' to refer to materials with dimensions below $1 \mu \mathrm{m}$. In fact, the plethora of NP compositions and architectures reported in the literature has led to the term 'nanoParticle Zoo' being coined to describe the current state of the field (Moore et al. 2015). The nanoParticle Zoo contains different materials [gold (Adnan et al. 2016), carbon (Wanga and $\mathrm{Hu}$ 2014), quantum dots (Alivisatos 1996), silica (Moore et al. 2015), liposomes (Chang and Yeh 2012), iron oxide (Danhier et al. 2010), etc.] with different shapes [spheres (Wang et al. 2011a), rods (Yasun et al. 2015), plates (Cai and Lin 2014), cages (Chen et al. 2007), stars (Nehl et al. 2006), worms (Park et al. 2008), onions (Bartolome et al. 2015), etc.] and different properties [plasmonic (Lukianova-Hleb et al. 2012), fluorescent (Wolfbeis 2015), magnetic (Lee et al. 2010), etc.], an array of materials that is challenging to categorise. In light of this scenario, we liken this current situation to that of the original Particle Zoo: the scenario faced by physicists in the middle of the twentieth century when new elementary particles were discovered with such regularity, thus requiring classification, that Wolfgang Pauli was prompted to supposedly remark "Had I foreseen this, I would have gone into botany".

Dye-doped silica NPs (DDSNs) have emerged as a potential fluorescent nanoprobe capable of being adapted to a multitude of bioapplications that offer fluorescent capabilities comparable to heavy metal QDs (which are generally classified as toxic). DDSN synthesis is straightforward and versatile enough to allow for different dyes and drugs to be housed in the silica matrix while still allowing for control over the resulting NP size and shape. In addition, their adaptable and well-understood chemistry allows a simple and straightforward modification of the NP surface chemistry to provide reactive sites for further functionalisation (Bagwe et al. 2006; Nooney et al. 2012). Indeed, other classes of NP are often coated with a thin layer of silica to improve NP biocompatibility and to provide a flexible platform upon which to conduct further reactions (Ma et al. 2014; Kobayashi et al. 2003; Gnanasammandhan et al. 2016). Furthermore, silica also does not 
absorb visible light and doping the silica matrix, embedded with photoactive molecules (either internally or bound on the NP surface), allows the NPs to exhibit photophysical properties similar to those of the chromophores (Yan et al. 2007; Bae et al. 2012). From a practical perspective, DDSNs exhibit a high density and can therefore be easily isolated into an aggregated pellet via centrifugation (Wang et al. 2008; Bae et al. 2012). The aggregated mass can be separated from the supernatant easily and ultrasonication is then usually required to effectively re-disperse back to their original monodispersed state (Bagwe et al. 2006).

This review discusses the advances in the field of DDSN in bioapplications and is primarily focused on microporous silica. Micropores are less than $2 \mathrm{~nm}$ in size (McCusker et al. 2001) and we have intentionally avoided referring to mesoporous silica, which exhibit $2-50 \mathrm{~nm}$ pores and are commonly used as a drug delivery nanomaterial ( $\mathrm{Li}$ et al. 2012). We begin with a thorough overview of the two common routes of silica NP synthesis: Stöber and reverse microemulsion methods. The process of dye-doping is then discussed, followed by surface functionalisation strategies and how DDSN surface chemistry influences colloidal stability. The developments in the use of DDSNs in biosensing, bioimaging and drug delivery are then presented, and are predominantly focused on cancer research. Importantly, the impact of the protein corona on understanding DDSN interactions with biological systems is also discussed and its implications in in vitro and in vivo studies are highlighted. This article concludes with a discussion on silica NP toxicity and how a lack of widely accepted approaches to nanotoxicology can result in conflicting experimental outcomes.

\section{Silica nanoparticle synthesis}

Silica NPs are usually generated using the sol-gel method and involves the hydrolysis, and subsequent condensation, of alkoxide precursors via acid or base catalysis. Tetraethyl orthosilicate (TEOS) is typically employed as a silica precursor. Base catalysis of silica precursors with ammonia is the most commonly used approach for NP generation (Fig. 1).

The most widely used synthetic strategies are the Stöber method and the reverse microemulsion method, both of which have their own advantages and disadvantages. As

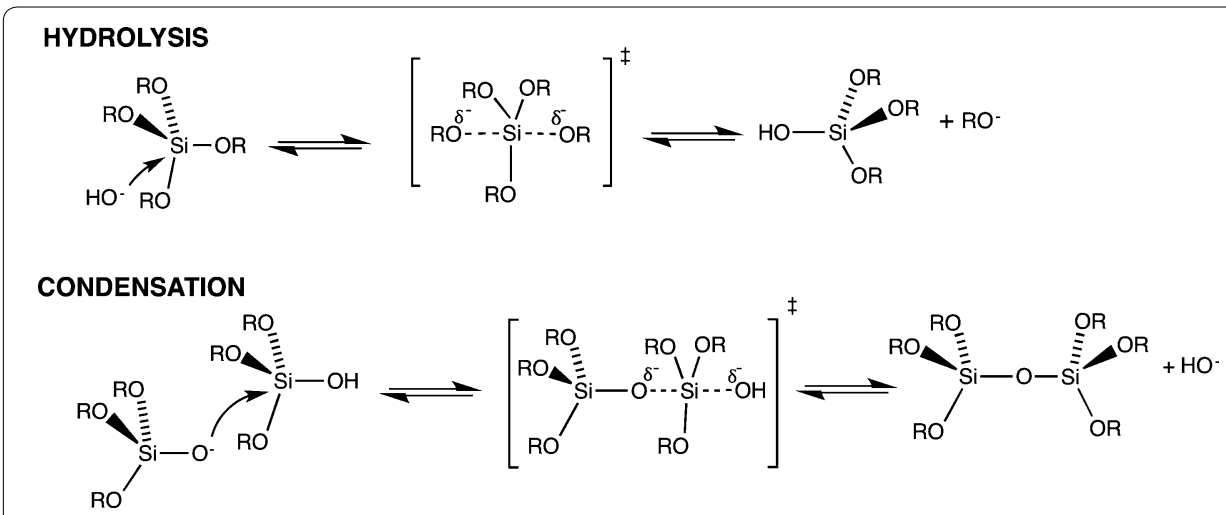

Fig. 1 Base-catalysed hydrolysis/condensation of silica precursors. Tetraethyl orthosilicate (TEOS) is the most commonly used precursor for generating silica nanoparticles 
means of creating luminescent NPs, dyes are typically functionalised with trialkoxysilanes such that they can be incorporated in the silica matrix during particle formation alongside TEOS, and this doping process can influence the resultant NP size (Nooney et al. 2012). Covalent attachment of dyes reduces dye leaching from the NP in comparison to when dyes are physically entrapped in the silica matrix, but it should be noted that covalent attachment does not completely prevent leaching as silica NPs can degrade in biological conditions, which is a feature frequently overlooked by researchers (Mahon et al. 2012b).

\section{Stöber method}

The Stöber method, first described in the 1960s (Stöber et al. 1968), involves the hydrolysis and condensation of a silica alkoxide precursor, typically TEOS, in a water/alcohol mixture catalysed by the addition of ammonium hydroxide (Fig. 2a) (Bonacchi et al. 2011). This synthetic route to control important parameters of the resultant NPs, such as size, porosity and colloidal stability by controlling a range of synthetic parameters such as precursor concentration (Huang and Pemberton 2010), reaction time (Yokoi et al. 2009), temperature (Huang and Pemberton 2010), stirring conditions (Yokoi et al. 2009) and the potential use of multistep regrowth procedures for controlled NP growth (Hartlen et al. 2008). Notable advantages of this method are that it is straightforward to perform, it produces particles in the $50 \mathrm{~nm}-2 \mu \mathrm{m}$ range and it can be upscaled. Therefore, the Stöber approach is widely used for multiple applications and when producing silica NPs for commercial applications. However, precise control of NP monodispersity and morphology can be problematic, with $<30 \mathrm{~nm}$ diameter NPs being challenging to synthesise (Wang et al. 2011a). The mechanism for NP formation remains unclear, with two models favoured by the scientific community (Korzeniowska et al. 2013). The first, proposed by LaMer and Dinegar described a 'monomer addition' situation where two distinct steps occur during synthesis: an initial burst of nucleation to form primary particles, followed by particle growth due to the addition of hydrolysed monomers to the primary particle surface (LaMer and Dinegar 1950; Matsoukas and Gulari 1989). The second model suggested by Bogush and Zukoski (1992) describes the continuous nucleation during the reaction where of primary sub-particles 'aggregate' into larger particles (Ab Rahman and Padavettan 2012).

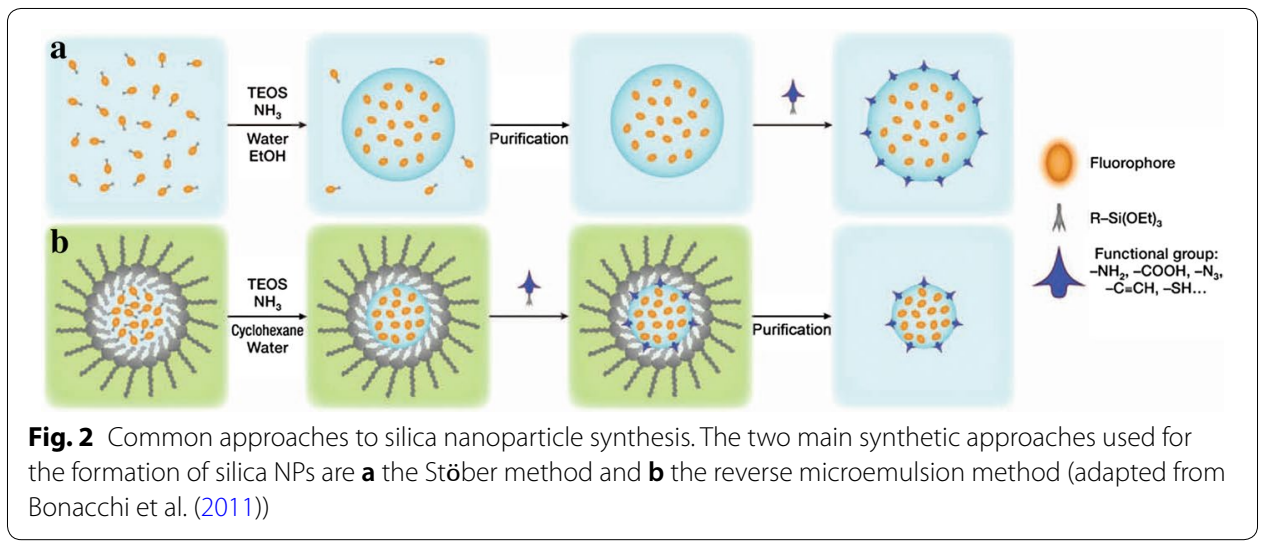


Variations on the Stöber method have also been reported. Wiesner and co-workers reported a modified Stöber method that first involved the formation of dye-loaded 'seeds' that were later covered by a denser silica network to give a fluorescent core-shell configuration (Burns et al. 2006; Ow et al. 2005). The shell protects the dye-loaded core from interactions with the surrounding solvent, thus enhancing particle photostability and brightness. These NPs were dubbed Cornell dots (C-dots). A second generation of silica NPs, termed Cornell prime dots ( $\mathrm{C}^{\prime}$-dots), have since been developed and involves water-based synthesis of sub-10 nm polyethylene glycol (PEG)-coated silica NPs (Ma et al. 2015). Using tetramethyl orthosilicate (TMOS) as a precursor, NP growth could be controlled by a number of factors such as the introduction of silane-functionalised PEG to the NP surface to cap particle growth. This in turn provided a functional surface supporting NP stability through steric interactions. In addition, the inclusion of an aluminium alkoxide precursor into the NP core enhanced the quantum yield of the encapsulated near-infrared dyes. C-dots and $\mathrm{C}^{\prime}$-dots have reported positive results from human clinical trial trials for in vivo tumour imaging (Phillips et al. 2014).

\section{Reverse microemulsion method}

The second method of silica NP formation is the water-in-oil reverse microemulsion (Fig. 2b) (Bonacchi et al. 2011). Here, surfactant-stabilised water droplets are dispersed in an oil phase to create micelles that serve as a 'nanoreactor' for the confined nucleation and growth of silica NPs. TEOS diffuses into the water phase (which also contains ammonia catalyst), and undergoes hydrolysis and condensation. Micelles collide and exchange nuclei and/or hydrolysed monomers, thus promoting NP growth (Finnie et al. 2007). The factors that govern the resultant NP include the water-to-surfactant ratio, concentration of ammonium hydroxide, and concentration of TEOS (Arriagada and Osseo-Asare 1999b). Bagwe et al. (2004) conducted a thorough study of these parameters to examine how they can influence size and polydispersity of $\left[\mathrm{Ru}(\mathrm{bpy})_{3}\right]^{2+}$-doped NPs. One particular finding involved the choice of surfactants of different natures (e.g. linear or branched, with or without charge) to show that micelle structure and rigidity can govern their collision dynamics, thus controlling the nucleation and growth mechanism in a given water-in-oil system. Consequently, NPs of different sizes were produced. The reverse microemulsion method has been heavily studied by Arriagada and Osseo-Asare $(1995,1999 b)$ with one their important works being the development of a statistical nucleation model to accurately describe silica NP formation in water-in-oil microemulsions (Arriagada and Osseo-Asare 1999a). Under similar synthesis conditions, the use of fluorescein isothiocyanate (FITC) produced larger NPs than fluorescein succinimidyl ester-doped particles. It was thought that due to fluorescein succinimidyl ester being less soluble in the water nanodroplets of the microemulsion, the supersaturation concentration was lowered.

This led to an increase in the number of nucleating sites, which in turn led to the formation of smaller NPs. In general, smaller monodisperse NPs (approx. 15-50 nm) can be produced using the reverse microemulsion method in comparison to traditional Stöber approaches. However, this synthetic approach is limited by the excessive use of organic solvent and surfactants (which can remain on the NP surface following purification). The need for excessive purification and solvent-cost therefore limits upscaling. 
Table 1 The advantages and disadvantages of the Stöber and reverse microemulsion method

\begin{tabular}{|c|c|c|c|}
\hline \multicolumn{2}{|l|}{ Stöber } & \multicolumn{2}{|l|}{ Reverse microemulsion } \\
\hline Advantages & Disadvantages & Advantages & Disadvantages \\
\hline $\begin{array}{l}\text { Water-based synthesis } \\
\text { possible }\end{array}$ & $\begin{array}{l}\text { Greater NP polydispersity } \\
\text { than reverse microemul- } \\
\text { sion }\end{array}$ & Precise control of NP size & $\begin{array}{l}\text { Excessive use of organic } \\
\text { solvents }\end{array}$ \\
\hline Surfactant-free & $\begin{array}{l}\text { Challenging to produce } \\
\text { sub-30 nm NPs }\end{array}$ & Small NPs (20-200 nm) & $\begin{array}{l}\text { Residual surfactant on } \\
\text { NP surface following } \\
\text { purification }\end{array}$ \\
\hline $\begin{array}{l}\text { Favoured for upscaling } \\
\text { (gram scale) }\end{array}$ & & & $\begin{array}{l}\text { Not suited to upscaling } \\
\text { (milligramme scale) }\end{array}$ \\
\hline
\end{tabular}

The reverse microemulsion system is well-suited for preparing other nanomaterials with silica shells. Creating a silica shell around quantum dot cores, for example, is used to offset the cytotoxic effects attributed to their heavy metal composition, thereby improving their biocompatibility and usefulness in bioimaging (Montalti et al. 2014). Creating a layer of silica around such NPs also lends itself to easier functionalisation with organosilanes bearing reactive functional groups (Wegner and Hildebrandt 2015). The main advantages and disadvantages of the Stöber and reverse microemulsion methods are listed in Table 1.

\section{Other methods}

The direct micelle-assisted method is another method that has grown in popularity as it allows for one-pot synthesis of PEGylated DDSNs, thus eliminating the need for later surface functionalisation (Montalti et al. 2014). Pluronic 127, a tri-block PEG-PPOPEG (poly(ethylene-glycol)-poly(propylene oxide)-poly(ethylene glycol)) is used as high molecular weight surfactant to form micelles. The relatively polar PPO inner core of the micelle allows lipophilic TEOS to be used as a precursor. Particle synthesis is then acid catalysed, using hydrochloric acid (Liu et al. 2009; Huo et al. 2006) or acetic acid (Rampazzo et al. 2012), and particle growth is confined to the micellar environment. The hydrophilic PEG moieties are directed outwards from the NP surface and ultimately results in silica-PEG core-shell architecture. By derivatising dyes with alkoxysilanes, it is then possible to covalently dope fluorophores into the particle core. Silica NPs generated using the direct micelle-assisted method have since been dubbed PLuS NPs (Pluronic silica NPs) (Pedone et al. 2013).

Dual-phase approaches for controlling the growth of small (10-100 nm) NPs have recently come to prominence (Wang et al. 2011a; Hristov et al. 2015a). An upper organic phase containing TEOS controls the transfer of the precursor to a catalyst-containing aqueous phase where NPs are grown and leads to greater control of NP size. Hristov et al. (2015a), for example, used organic solvents of different polarity (ethanol, isopropanol, 1-butanol, 1-hexanol and cyclohexane) to control the transfer rate of TEOS monomers to the aqueous phase. This in turn governed the rate of hydrolysed monomer generation and the controlled production of NPs in the $10-100 \mathrm{~nm}$ range. 
Organically modified silica (ORMOSIL) NPs are another class of silica NPs that typically employ alkyl-substituted silicon alkoxides as precursors to modify the silica network, which facilitates the incorporation hydrophobic materials into the NP (e.g. dyes or chemotherapeutics). For example, the use of phenyltrimethoxysilane and methyltrimethoxysilane as precursors for the entrapment of porphyrins, and subsequently allowed for the development of a dual-dye sensor for intracellular dissolved oxygen detection (Koo et al. 2004). ORMOSIL NPs have since become used in vivo imaging and drug delivery applications (Ohulchanskyy et al. 2007; Kumar et al. 2010; Roy et al. 2014; Nagesetti and McGoron 2016).

Though less popular, non-spherical silica nanomaterials can also be generated. Imhof and co-workers have demonstrated the possibility to generate bullet-like silica rods through a one-pot synthesis containing ethanol, water, sodium citrate and ammonia in a solution of polyvinylpyrrolidone (PVP) in pentanol (Kuijk et al. 2011). Rods with diameter 200-300 nm and lengths of up to $10 \mu \mathrm{m}$ could be produced using this method. Due to the straightforward chemistry of silica, it was also possible to label the rods with fluorescent dyes. It was later possible to etch rod-like particles into cone-like particles using ammonium hydroxide (Hagemans et al. 2016). This work was further extended to show that spheres and rods could be etched into dumbbells and biconcave silica rings sandwiched between two silica spheres (Hagemans et al. 2017). Silica 'matchstick' colloids have also been reported (Longbottom et al. 2015).

\section{Dye encapsulation}

Dye molecules can be incorporated into the silica matrix by two means: physical entrapment or covalent attachment. Positively charged dyes, such as tris(bipyridine) ruthenium(II) chloride, $\left[\mathrm{Ru}(\mathrm{bpy})_{3}\right]^{2+}$, are well-suited for physical entrapment into the negatively charged silica network, but covalent linking is preferred because it minimises dye leakage from the NP over time. The first report of covalent attachment of dye molecules into the matrix of silica NPs was by van Blaaderen and Vrij in 1992 (Van Blaaderen and Vrij 1992). This involved the coupling of FITC to an oxysilane coupling agent (3-aminopropyl) triethoxysilane (APTES), via a thiourea bond and the controllable incorporation of the FITC-APTES reaction product into the silica structure.

In general, obtaining NPs of maximal brightness is desirable as it enables high sensitivity and the detection of low concentration analytes. The choice of fluorophore is central to achieving this goal and is application dependent. For example, one must consider the fluorophore's excitation and emission wavelengths. The dyes used should ideally be readable by common laser lines to facilitate their use in biomedical diagnostics and bioimaging. For this reason, fluorescein is often employed as it can be excited at $488 \mathrm{~nm}$ by standard argon lasers employed in confocal microscopy and flow cytometry. Near infrared (NIR) labels are advantageous for use in applications incorporating whole blood as blood exhibits a weak absorption band. This is important as it reduces the need for sample filtering and allows for more straightforward biosensor design (Korzeniowska et al. 2013). In vivo imaging is also possible using near infrared (NIR) dyes. For example, Wiesner's C-dots incorporate Cy5, in conjunction with PET probes, for non-invasive NP imaging/tracking (Phillips et al. 2014; Benezra et al. 2011). However, commercially available near infrared dyes are on the order of $\$ 200-\$ 300 / \mathrm{mg}$ (Ma 


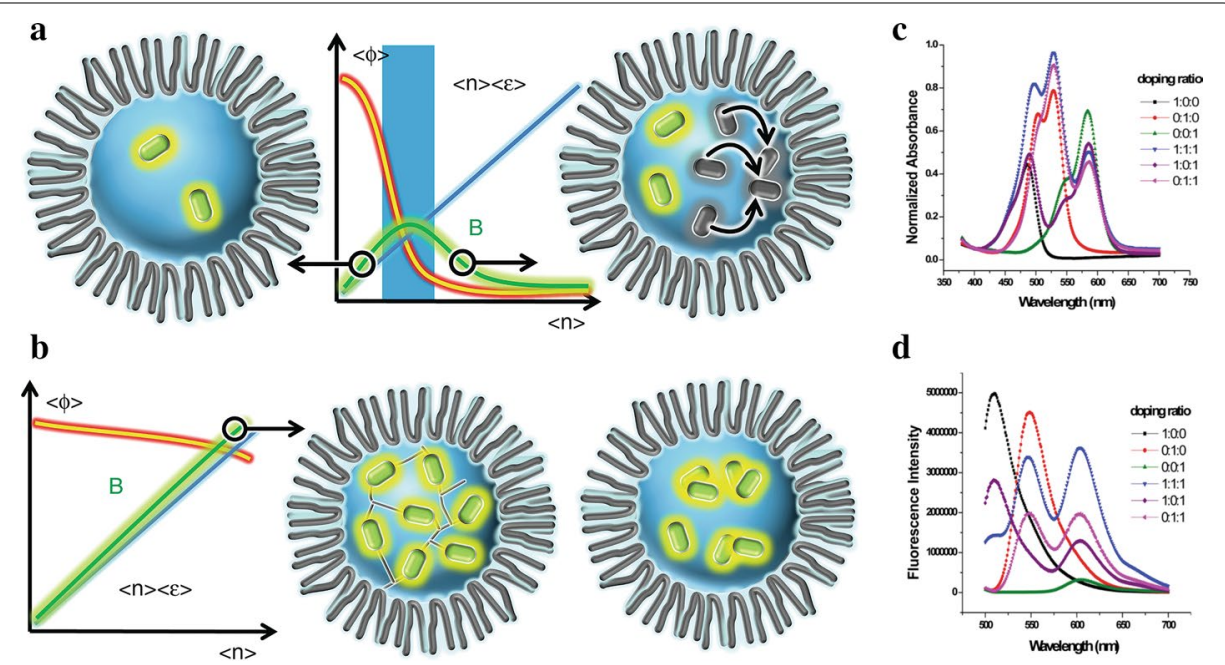

Fig. 3 Optimising dye loading is crucial to achieving maximal NP brightness. a (left) A low number, $n$, of fluorophores present in the NP maximises quantum yield, $\varphi$, but NP brightness, B, is low. a (right) A high concentration of fluorophores can lead to FRET/self-quenching and result in a low brightness NP. The optimal loading window in shown in blue. $\mathbf{b}$ Left: ideally, self-quenching and FRET can be minimised by controlling dye distribution or, $\mathbf{b}$ (right) using molecules with large Stokes shift such their proximity is not problematic. c Absorbance spectrum of silica NPs doped with different FITC:Rhodamine6G:ROX ratios. d Different dye-doping ratios could generate silica NPs with different fluorescent signatures ( $\mathbf{a}$, $\mathbf{b}$ adapted from Battistelli et al. (2016); c, d adapted from Wang and Tan (2006))

et al. 2015) and demonstrates that the choice of dye can be cost-dependent as well as application-dependent.

Creating NPs with optimal brightness is another point of consideration when developing DDSNs. This is largely associated with the concentration of dye molecules bound inside the silica matrix (Fig. 3) (Battistelli et al. 2016). If too few fluorophores are encapsulated inside the core then the resultant NP may insufficiently bright. Interestingly if too many dyes are densely packed inside the silica core, the overall brightness might be reduced through self-quenching (Fig. 3a, b). The reduced NP fluorescence intensity due to dye overloading can be attributed to Förster resonance energy transfer (FRET), fluorophore aggregation and inner filter effects (Miletto et al. 2012; Battistelli et al. 2016).

Commonly used dyes in biological applications, such as fluorescein, exhibit large excitation and emission overlap (small Stokes shift) and if situated in close proximity to one another FRET can occur. If two of the same dye molecule are in proximity of each other and experience FRET, this phenomenon is known as homo-FRET or self-quenching. Studies have therefore focused on the optimisation of dye loading into silica NPs (Imhof et al. 1999; Nooney et al. 2009). Nooney et al. (2009) addressed this issue by developing a dye loading model for the optimisation NP fluorescence in relation to concentration of NIR-664 succinimidyl ester loading into the NP core. One way of circumventing the issue of homo-FRET is by choosing fluorophores with large Stokes shift such that their excitation and emission wavelength do not greatly overlap (Herz et al. 2009). For example, Hell and co-workers used large Stokes shift dyes with greater than $140 \mathrm{~nm}$ absorbance/emission maxima separation for stimulated emission depletion (STED) microscopy for cellular labelling (Butkevich et al. 2017). However, 
such dyes are usually expensive in comparison to common fluorophores and are yet to gain widespread use.

Despite these considerations, the issue of self-quenching is not always regarded as a disadvantage when it comes to formulating DDSNs. For example, Moore et al. used fluorescein-overloaded silica NPs to improve immunoassay fluorescent signals. The dye was intentionally overloaded into silica NPs such that self-quenching reduced NP brightness, but following antigen recognition the NPs were degraded using basic buffer. This led to the release of the fluorescent cargo from the NPs and the liberation of the fluorophores from self-quenching. Coupled with the fluorescein's increased brightness in basic conditions, the large quantity of free dye exhibited an order of magnitude increase in fluorescent signal compared to traditionally used NPs loaded with optimum quantities of dye (Moore et al. 2017).

Tan et al. exploited FRET by ratiometrically encapsulating FITC, Rhodamine 6G and ROX into silica NPs to create silica NPs with specific fluorescence signatures under single wavelength excitation (Wang and Tan 2006). The use of dyes with overlapping excitation and emission wavelengths allowed for large Stokes shifts to be produced. For example, doping the three fluorophores in 1:1:1 ratio made it possible to use FITC excitation $(490 \mathrm{~nm})$ to produce emission around $608 \mathrm{~nm}$ (i.e. ROX emission) caused by the donor-acceptor cascade between the individual dye absorbance and emission bands. The generation of multicolour silica NPs made them suitable for multiplex analysis (Fig. 3c, d).

Doping silica NPs with fluorescent organic molecules has also been shown to be useful in elucidating the structural integrity of the internal particle matrix over time. Dawson and co-workers adsorbed thioflavin T into Stöber-synthesised NPs and exploited the rotor-based fluorescence of the molecule. Intact silica NPs restricted the rotational freedom of thioflavin $\mathrm{T}$ which results in fluorescence spectrum red-shifting. The molecule regained rotational freedom following NP degradation and resulted in a reduction of fluorescence emission of up to three orders of magnitude, thus serving as an indicator of matrix integrity. The use of thioflavin T-doped silica NPs was then used to track NP structural integrity in in vitro experiments (Meder et al. 2016).

Computational approaches to analysing surface chemistry can also be employed as tools to achieve a better understanding of synthesised NPs. For example, interactions of salts (de Lara et al. 2012, 2015) and polymers (Hong and Panagiotopoulos 2012) with the surface of silica can be modelled, as well as interactions with proteins (Sun et al. 2014). The outcome of controlled approaches to silica NP synthesis has also been possible to verify using computation simulations (Suzuki et al. 2010).

Overall, silica NPs are a flexible platform upon which fluorescent particles can be produced without great synthetic efforts. However, it is important to consider the intended application of the NPs and the overall cost of production prior to experimentation since this will determine the choice of dye and the most appropriate synthetic route. Covalent binding of dyes inside the silica matrix will retain fluorophores inside the particle core longer than those physically entrapped (Korzeniowska et al. 2013). Particle dissolution occurs in aqueous media and can result in dye leaching, which means storing NPs in solvents like ethanol is preferred. DDSNs can even be stored in gels for longer-term storage (Giovannini et al. 2017). Dye leaching can be monitored using simple techniques 
like dialysis, whereby free dye can be separated from NPs and then optically quantified. Transmission electron microscopy can also be used to examine dissolution-induced NP hollowing/etching (Giovannini et al. 2018a). Stabilising DDSNs against dissolution in aqueous media is therefore advised before biological experimentation as the release of free dye can lead to misleading experimental outcomes (Mahon et al. 2012b).

\section{Surface chemistry}

\section{Post-synthesis NP coating}

An unmodified and pristine silica NP is inherently negatively charged due to surface hydroxyls, but other more reactive groups are usually added to the NP exterior following synthesis. Functional groups such as thiols (Claesson and Philipse 2007), epoxy (Zhang et al. 2009), vinyl (Sharma et al. 2004) and amines (Bagwe et al. 2006) can be introduced by adding alkoxysilane derivatives (Wang et al. 2008; Malba et al. 2014). These functional groups aim to render the surface neutral or introduce charge, which in turns provide advantages such as increased biocompatibility, supramolecular recognition or simply serve as reactive sites for future linker or biomolecule attachment.

Amine modification is widely used because it allows for later reactions with aldehyde- or NHS ester-functionalised biomolecules, linkers, or dyes. The most popular, inexpensive and commercially available reagents for amine surface treatment are aminopropyltrimethoxysilane (APTMS) and aminopropyltriethoxysilane (APTES). NP surface treatment with APTES can be performed in situ where the aminoalkoxysilane is introduced in the later stages of polymerisation within the Stöber process once the nuclei are formed (An et al. 2007). In such cases the aminoalkoxysilane reagent is typically used in excess and any unreacted molecules are removed during the purification steps.

However, despite being considered efficient, this strategy can lead to formation of disordered multi-layers since the degree of surface modification depends on multiple factors such as solvent used, water content, temperature, reaction time, etc.

Additionally, there are several possible modes of attachment/association of APTES with a silica surface caused mainly by hydrogen bonding, other non-covalent interactions or vertical polymerisation. In addition, homogenous amine modification of the silica surface is generally challenging because aminoalkoxysilanes in solution are prone to uncontrolled polymerisation (Fig. 4A) (Zhu et al. 2012; Smith and Chen 2008). To achieve uniform amine monolayers it is therefore necessary to carefully control reaction conditions (Schulz et al. 2011) and the amount of APTES used (Hristov et al. 2015b). An interesting study by Chen and Smith compared commercially available short- and long-chained aminoalkoxysilane derivatives (5, 8, $10 \AA$ chain length) and examined their ability to be removed from the silica NP surface through intra or intermolecular selfcleavage (Fig. 4B, C) (Smith and Chen 2008). The authors found that the amine functionality can be lost on silica surface over time because the primary amine in the propyl position of APTMS and APTES is capable of coordinating with silicon atoms either intramolecularly (via the formation of a five-membered cyclical intermediate) or intermolecularly and catalyses the cleavage of the siloxane bonds via hydrolysis (Fig. 4B). However, $N$-(6-aminohexyl)aminomethyltriethoxysilane-derived surfaces were more resistant to hydrolysis and produced stable amine-functionalised silica surfaces due to longer chain length being incapable of intramolecular coordination with silicon atoms 

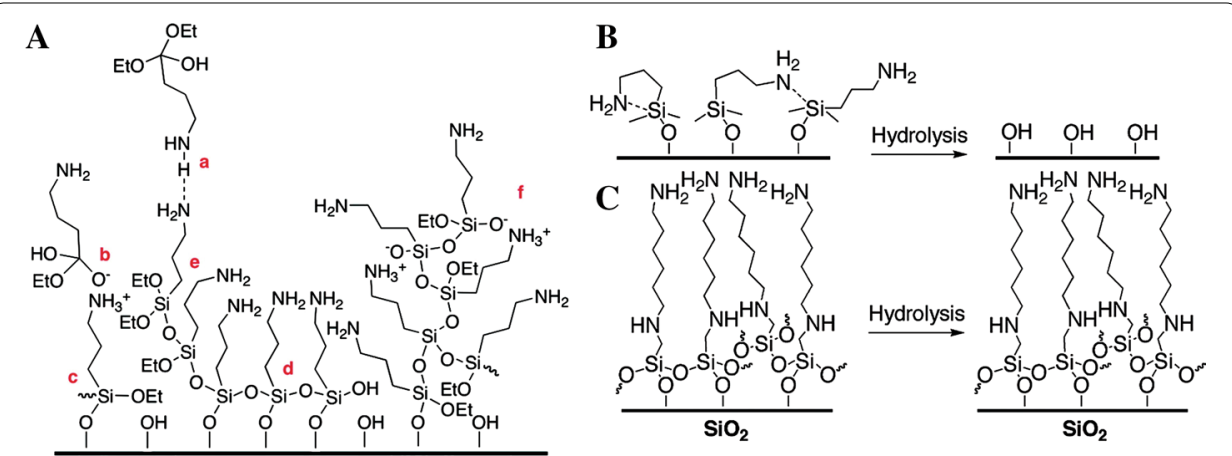

Fig. 4 Post-coating unmodified -OH bearing silica NPs is commonly done using siloxanes with reactive amines. A However, such coatings are not usually homogenous and individual silane molecules can interact through (a) hydrogen bonding, (b) electrostatic attraction, (c) covalent bonding with surface, (d) horizontal, and (e) vertical polymerisation with neighbouring silanes. (f) Oligomeric/polymeric silanes can also react/ interact with functionalities present at the interface. $\mathbf{B}$ When the reactive amine is in the propyl position it can coordinate intra- or intermolecularly with silicon atoms and catalyse the cleavage of the siloxane bond via hydrolysis, thus eventually resulting in the loss of amine groups from the silica surface. CThe longer chains of $\mathrm{N}$-(6-aminohexyl)aminomethyltriethoxysilane are not capable of self-cleave and give stable amine layers on silica surfaces (A: adapted from Zhu et al. (2012); B, C: adapted from Smith and Chen (2008))

(Fig. 4c), thus suggesting that longer chained aminoalkoxysilanes may retain amine functionality on silica for longer.

Successful amine modification is usually confirmed by the change in zeta potential, but does not quantify the functional groups surface density (this is important for toxicological studies as amine-modified materials can increase cytotoxicity) (Shahabi et al. 2015). Acid-base titration (Jung et al. 2012), colorimetric or fluorescent reactions (Chen and Zhang 2011; Noel et al. 2011), thermogravimetric analysis (Lu 2013), or solution (Hristov et al. 2015b; Kunc et al. 2018) or solid-state NMR (Sun et al. 2019) are examples of quantitative methods used to accurately characterise the content of amine and other functional groups on silica NPs surface.

It should be noted that these methods vary in their sensitivity and the ranges of applicability. The choice of method will depend on given the functional group. Reactive chemical groups such as amines or thiols can be quantified by chemical assays, these however require extensive optimisation since their output may not be quantitative (Sun et al. 2019). Thermogravimetric analysis is typically less sensitive and hence more applicable for the analysis of thick polymer coatings. This is because for silica NPs, TGA is further complicated by the interference arising from the co-condensation of silanol hydroxyls occurring throughout the whole temperature gradient. Solidstate NMR is limited by the high amount of material and the long measurement time required for analysis of less abundant ${ }^{13} \mathrm{C}$. This may be overcome by labelling by isotope labelling, if applicable. For silica NPs, solution NMR appears as the most facile method for total quantification of functional groups, however, this depends on the functional group solubility aqueous media (Kunc et al. 2018).

Post-synthesis coatings of silica NPs with amine-reactive moieties can promote colloidal stability via electrostatic repulsion, but it should be noted that such stability is dependent on, for example, $\mathrm{pH}$. Amine groups on the NP surface can be protonated $\left(-\mathrm{NH}_{3}{ }^{+}\right)$positively charged in physiological $\mathrm{pH}$, whereas the silica matrix is 
negatively charged in such conditions (Bagwe et al. 2006). Consequently, a reduction in the surface charge of the NP occurs and means NP-NP repulsion is reduced, thus giving rises to aggregation. A notable study by Bagwe et al. (2006) showed how a combination of negatively charged 'inert' phosphonate groups and 'reactive' amine groups can be combined on the surface of RuBpy-doped silica NPs to reduce particle aggregation, while also still providing the reactive sites needed for later functionalisation. 'Bipodal' bis amine[3-(triethoxysilyl) propyl] has also been proposed as an alternative to traditional 'monopodal' APTMS as the former produces more colloidally stable amine-coated silica NPs (Kelleher et al. 2015). Wang et al. demonstrated that silica NPs can also be used as a platform for catalysis and was facilitated by the use of amino-functionalised colloids. The amino groups served as a template upon which a series of intermediate functionalisations could be carried out and ultimately yield TEMPO-coated silica NPs capable of benzylic alcohol oxidation (Liu et al. 2015).

Overall, the use of common aminoalkoxysilanes like APTES or APTMS allows for silica NPs to be used as a platform upon which advanced functional materials can be designed. Nonetheless, as highlighted by this section, researchers should carefully consider the coating strategy when attempting to generate amine-functionalised silica NPs. Other examples in the literature that summarise silica NP synthesis and functionalisation can be found elsewhere (Wang et al. 2013; Liberman et al. 2014; Montalti et al. 2014).

\section{Bioconjugation strategies}

Bioconjugation in the context of nanomaterials means attachment of biomolecules (e.g. proteins, enzymes, oligonucleotides, etc.) onto the surface of NPs. Usually it can be performed by two methods; passive absorption or covalent attachment (Gubala et al. 2018b). Passive adsorption is straightforward to conduct, cost efficient and leads to the adsorption of protein layers surrounding the NP surface. However, because the biomolecules are not covalently attached they can be removed and replaced by other biomolecules that exhibit higher affinity towards the NP surface. For proteins, the most frequently used biomolecule for bioconjugation, changes in conformation or local $\mathrm{pH}$ influence a protein's isoelectric point and subsequently its electrostatic interactions with the NP surface, which can then cause desorption of biomolecule (Korzeniowska et al. 2013). Vertegel and co-workers (2004) also studied how particle size influences the structure and activity of protein adsorbed to $4 \mathrm{~nm}, 20 \mathrm{~nm}$ and $100 \mathrm{~nm}$ silica NPs. It was shown that adsorbing lysozyme to increasingly larger silica NPs leads to increased $\alpha$-helix structural loss, thus causing reduced enzymatic activity. The authors suggested that protein-NP interactions increased on the less curved surfaces of the larger NPs and resulted in secondary structural loss. The adsorption of cytochrome $c$ to silica NPs of different sizes also followed a similar trend (Shang et al. 2009).

The more widely used method of functionalising silica NPs with biomolecules is through covalent bond formation, and is typically facilitated by a linker molecule (Korzeniowska et al. 2013; Moore et al. 2015). The covalent approach is typically a twostep process where, firstly, the linker is introduced to the NP surface and, secondly, the biomolecule of interest is bound to the NP via the linker. The linker also serves as a spacer between the biomolecule and the NP surface, thus allowing the biomolecule to 
experience greater solvation and reduces potential for misfolding due to NP-biomolecule interactions (Korzeniowska et al. 2013; Hristov et al. 2015b). It also allows for desirable reactive groups to be imparted to the NP surface prior to the introduction of the biomolecule.

Linkers are generally used to bind to specific amino acid residues of an antibody. For example, there are approximately 83 lysine residues per whole IgG biomolecule (Tan et al. 2008) and these primary amines can be used as reactive sites for covalent attachment using ethyl(dimethylaminopropyl) carbodiimide EDC (EDC)/ $N$-hydroxysuccinimide (NHS) chemistry (Moore et al. 2015; Kunc et al. 2019). While the use of EDC/NHS coupling is very popular for bioconjugation, it should be noted that due to the presence of amine groups throughout the protein it is therefore not possible to control the orientation of the antibody upon attachment to the NP surface, and can ultimately result in binding epitopes that are incapable of recognising their target.

'Click' chemistry is an increasingly popular form of bioconjugation that involves reactions of high stereospecificity and high efficiency. In particular, copper-free strain promoted cycloaddition 'click' reactions between strained alkynes and azides are well-suited for biological applications as they do not require the use of potentially toxic, dye-quenching copper catalysts [which were employed in the original alkyne-azide cycloaddition reactions (Rostovtsev et al. 2002; Tornøe et al. 2002)]. One specific requirement for click chemistry is the necessity to site-specifically introduce click-reactive groups to the protein, which are typically inserted distal to the antigen-binding domain (Sivaram et al. 2018). However, once achieved, the antigen-binding site of the 'click'-bound antibodies can then become more accessible on the NP surface and, compared to traditional EDC/ NHS that produce randomly orientated antibodies, can improve the overall target-binding capacity of the protein-functionalised NPs. In addition, the use of 'click' reactions for bioconjugation (compared to traditional EDC/NHS) offers the possibility to reduce the quantity of antibody used per reaction due to high binding efficiencies (Sivaram et al. 2018).

The nature of the crosslinker has significant effect on the bioconjugation reaction. Crosslinkers can be either monovalent or multivalent in nature, and choosing the appropriate form depends on different factors like the availability of reactive groups on the NP surface, the potential for the linker to induce unwanted NP aggregation, linker commercial availability and the application the NPs are intended for. For example, Gubala et al. (2010) showed that, in comparison to monovalent linkers, using multivalent polyamidoamine (PAMAM) dendrimers for bioconjugation can be used to increase the number of binding sites on silica NPs for antibody coupling, and was particularly useful since the number of post-coated primary amines on the particle surface was restricted in order to maintain colloidal stability. The multiple binding sites afforded by PAMAM-coated silica NPs lead to greater number of antibodies being bound compared to monovalent glutaraldehyde and sulfo-SMCC. This work was later extended to show that increasing PAMAM multivalency, which allowed for greater number of antibodies per NP, leads to enhanced immunoassay kinetics when detecting C-reactive protein, a cardiovascular disease biomarker (Gubala et al. 2011). Multivalent carboxy-dextran has also shown to be a potential cost-effective alternative to multivalent PAMAM for bioconjugation of antibodies to DDNPs (Kunc et al. 2019). 
Polyethylene glycol is a linker molecule frequently used to functionalise NPs for drug delivery applications (Allen and Cullis 2013) and is rationalised by the strategy of improving the circulation half-life of NPs in vivo to exploit the enhanced permeability and retention (EPR) effect for localising in tumour sites (He et al. 2008). Controlling the density of PEG on the NP surfaces to form 'brush-like' conformations appears to be a crucial factor that determines the ability of NPs to evade immune cell uptake and to impart 'stealth' properties (Adumeau et al. 2017; Yang et al. 2014).

The polymer is commercially available in a variety of forms (short chained, polymeric or hyper-branched) and is highly hydrophilic, neutrally charged and biocompatible. It can also be bought with a wide range of functionalities and therefore allows for biomolecule attachment, thus combining 'stealth' and 'targeting' into a single colloidal system (Alric et al. 2016). A noteworthy example of dual stealth/targeting are Wiesner's sub$10 \mathrm{~nm}$ DDSNs that underwent human clinical trials and utilise PEG functionalised with targeting peptides (Phillips et al. 2014; Benezra et al. 2011). Interestingly, the mechanism of PEGylation of these small NPs (achieved using a PEG-silane) has been elucidated using fluorescence correlation and cross-correlation spectroscopy (Ma et al. 2016).

Firstly, the PEG-silane non-covalently associates with the silica surface (presumably through hydrogen bonding), followed by the accelerated condensation of the PEG-silane on the particle due to the molecule's proximity to the NP surface. This functionalisation mechanism allowed for the rapid transition between NPs stabilised through electrostatic repulsion to those stabilised through steric interactions, thus providing monomodal NPs. When NPs exhibited a low PEG density on NP surface, electrostatic stabilisation was greatly reduced and steric stabilisation was insufficient, which led to NP aggregation.

It should be noted that while the benefits of PEGylation are frequently described, others have reported that PEGylated particles can elicit PEG-specific immunoglobulin $M(\operatorname{IgM})$, which induces rapid elimination and liver uptake upon repeated doses of PEG-coated NPs. This has come to be known as the 'accelerated blood clearance' phenomenon (Fang et al. 2011; Ishida and Kiwada 2008).

Overall, while many reports describe the functionalisation of silica NPs with biomolecules as facile, it is in fact non-trivial and researchers need to carefully consider the bioconjugation chemistries used for attachment as it can affect biomolecule orientation on the NP surface (Saha et al. 2014; Jeong et al. 2017) and overall colloidal stability of functionalised NPs (Moore et al. 2015). It is therefore appropriate to evaluate bioconjugation strategies based on the biological performance of the resultant biofunctionalised NP. In doing so, the 'active surface' of the NP (i.e. the portion of the NP surface containing biomolecules capable to target recognition) can be elucidated and the bioconjugation strategy effectively scrutinised (Moore et al. 2015; Alric et al. 2016). For example, Herda et al. (2017) grafted antibody fragments to PEG-coated silica NPs and showed that only $3.5 \%$ of the randomly bound biomolecules exhibited favourable orientation for antigen recognition.

Extensive and informative reviews of strategies for bioconjugation can be found elsewhere and such focussed reading is beyond the scope of this more general review (Biju 2014; Sapsford et al. 2013). However, it should be acknowledged that 'Bioconjugate Techniques' by Hermanson (2013) is the cornerstone and general reference 


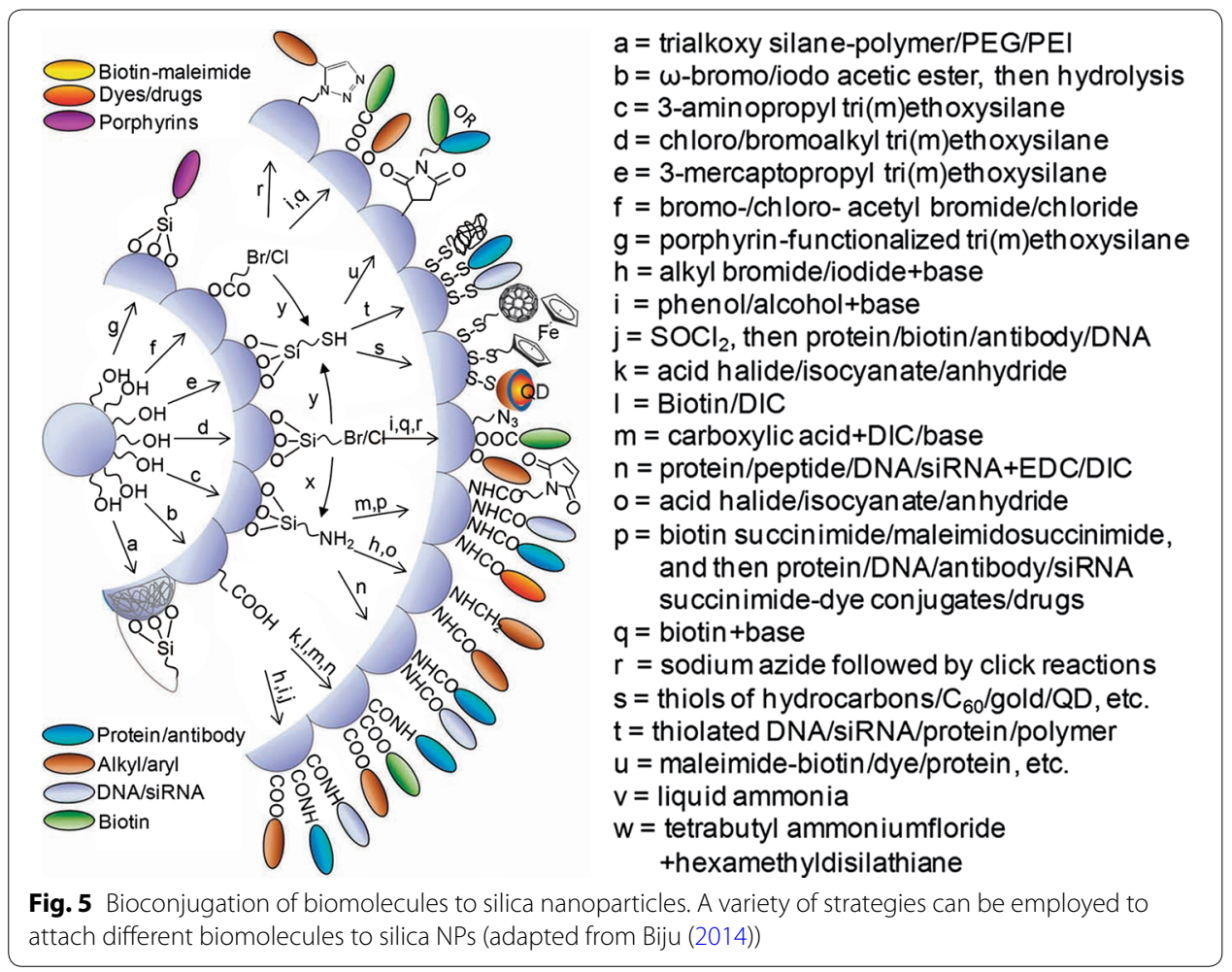

point in the field of bioconjugation. Figure 5 shows popular strategies of NP-biomolecule conjugation (Biju 2014). Indeed, it should also be noted that in the case of in vivo drug delivery the merits of using of NPs functionalised with targeting moieties remains a matter of debate (Cheng et al. 2012). The development of a NP with functionally active biomolecules on the surface has become more challenging than expected as the scientists working in the field encountered unforeseen challenges. In particular, the targeting molecule, such as an antibody or a ligand, must be functionalise in an optimal way to ensure that the binding site are available for binding and that they are not linked directly to the NP surface, but also that the targeting efficacy remains high also when the NP are exposed to a complex biological milieu (Nichols and Bae 2014; Salvati et al. 2013). The choice of a linker or a space between the NP and the biomolecule can affect the NP behaviour and targeting properties as the choice of the linker and the left can affect the ligand-binding properties of the functionalised nanomaterials (Hristov et al. 2015b). Despite the technical challenges, there is the need of an orthogonal set of methods that can be applied to characterise the newly synthesised nanomaterials but also to test whether the active ligand is biologically accessible and prone to interaction with the targeted molecule.

\section{Applications}

\section{Immunoassays and biosensors}

To date, the trusted immunoassay format for disease diagnosis in clinical setting is the enzyme-linked immunosorbent assay (ELISA) and is workhorse of the medical industry (Chatziharalambous et al. 2016; Tighe et al. 2015). However, due to emergence of NPs 
in biological research over the past two decades, researchers have focussed on exploiting the physicochemical properties afforded by these nanomaterials in order to achieve better signal-to-noise ratios, lower limits of detection, better sensitivity and faster testing times afforded by classical immunoanalytical methods (Farka et al. 2017). Indeed, the most successful example of these efforts is the gold NP-based pregnancy test for the detection of human chorionic gonadotropin in urine in less than $10 \mathrm{~min}$ (Gubala et al. 2012).

Variations to ELISA have been described using silica NPs whereby enzymes and targeting antibodies are co-immobilised on silica NPs. For example, Wu et al. (2009) attached horseradish peroxidase (HRP) and $\alpha$-fetoprotein antibodies on the surface of silica NPs and utilised the enzyme for signal transduction. Ke et al. also used dualfunctional silica NPs instead of traditionally used enzyme-labelled antibodies as reporter molecules. Amine-coated silica NPs were firstly functionalised with oxidised HRP via Schiff base chemistry and investigations into how the spacer length of the amino silane (i.e. the number of atoms between the NP surface and the reactive $\mathrm{NH}_{2}$ ) used during post-coating influenced the enzyme's binding were carried out. Three aminoalkoxysilanes of different space lengths (4, 7 or 10 atoms) were chosen and it was shown that the longest spacer bound the most HRP to the NP surface, which was attributed to a reduction in steric hindrance and easier access of the enzyme to the reactive site. Residual reactive amines on the NP surface were then reacted with oxidised dextran and the carbohydrate then served as a crosslinker for antibody attachment for the detection of hepatitis B surface antigen, with a detection limit 3 times lower than a commercial ELISA being reported (Ke et al. 2010).

Parallel approaches to ELISA using dye-labelled antibodies as probes that facilitate fluorescence-based assays are also common and are known as fluorescence-linked immunosorbent immunoassays (FLISA). However, these common organic dye labels are often prone to photobleaching (Gao et al. 2005) and when bound to antibodies the protein itself can induce chromophore quenching (Chen et al. 2010). The limitations of these probes have therefore led to the development of optically superior 'second generation' labels based on NPs such as quantum dots and dye-doped silica (Montón et al. 2009, 2012). Silica is particularly attractive due to its predictable and adaptable chemistry, and has led to derivatives of a number of commonly used organic dyes [such as fluorescein (Moore et al. 2017; Nooney et al. 2012), rhodamine (Dhir and Datta 2016) and cyanine (Nooney et al. 2015)] can be covalently bound inside the particle core or on the particle surface.

Rusling and co-workers used $\mathrm{Ru}$ (bpy)-doped silica NPs, prepared by the reverse microemulsion method as labels in an electrochemoluminescent sensor for the detection of prostate-specific antigen (Sardesai et al. 2009). The NPs were labelled with secondary antibodies in a sandwich assay format, with the oxidation of $\mathrm{Ru}$ (bpy) used to generate luminescence. This work has since been developed into an automated microfluidic array for the detection of four different prostate cancer biomarker proteins (Kadimisetty et al. 2015). Ruthenium-doped NPs have also been used as fluorescent labels in a microarray format for the detection of interleukin-6 (IL-6), a cytokine secreted by T-cells and macrophages during inflammation (Wu et al. 2008a). Capture antibodies bound IL-6 and secondary anti-IL6-coated NPs were used as fluorescent reporters. 
Producing fluorescent silica NPs is not limited to the use of traditional organic dyes. Wu et al. doped fluorescent carbon nanodots into silica NPs using a reverse microemulsion approach, and used these carbon-dot@silica NPs as optical reporter probes for the detection of $\alpha$-fetoprotein (AFP). The $45 \mathrm{~nm}$ NPs were functionalised with anti-AFP and were capable of capturing AFP in solution. FITC-labelled antibodies were then employed to sandwich AFP on the NP surface. The ratiometric signal between the carbon dots and FITC was used for signal transduction across a linear range of $0.317-35 \mu \mathrm{g} / \mathrm{dL}$ (Wu et al. 2016). The doping of europium into silica NPs has also been reported (Tagaya et al. 2011) and has been shown to be capable of facilitating HIV p24 antigen detection in clinical samples (Chunduri et al. 2017).

The use of broad spectrum antibiotics has lead to the rise of multi drug resistant bacteria and has meant DDSNs are also being used in assays for improved bacterial detection and identification. This is undoubtedly pertinent as multidrug resistant bacteria have arisen due to the Li and Xu used FITC-doped silica NPs in a sandwich assay format for the detection of Shigella flexneri, a bacillary dysentery endemic in countries like China, Bangladesh, Pakistan and Indonesia (Li and Xu 2009). Sun and co-workers (2015) have demonstrated the capability of detecting Salmonella pullorum using $45 \mathrm{~nm}$ silica NPs doped with C.I. Reactive Blue 21. This work was later extended to detect Salmonella pullorum and Salmonella gallinarum in powdered milk samples, with a total assay time of $1 \mathrm{~h}$ (Sun et al. 2016). An aggregation-based assay was demonstrated by Jayawardana and co-workers (2015) for the detection of the model bacteria Mycobacterium smegmatis. Japanese company Furukawa Electric has used commercialised a lateral flow assay incorporating fluorescent silica NPs, called Quartz Dots $(290 \mathrm{~nm})$ for the detection of different infectious microorganisms (Toriyama et al. 2015).

The current challenge for the development of Immunoassays and biosensors applications is to achieve reliable and stable nonoprobes that will allow good batch-to-batch reproducibility and optimal response in the fabricated device. Immunoassay research is dominated by gold nanoparticles (Hildebrandt and Tagit 2018), and other classes of nanomaterials like magnetic particles, photonic crystals and carbon dots are also increasingly used (Banerjee and Jaiswal 2018). However, DDSNs continue to be a highly useful probe due to their ability to be synthesised on large scales, their straightforward functionalisation and ability to be loaded with different fluorophores.

\section{Bioimaging}

In vitro

In the context of this review, in vitro bioimaging typically involves the incubation of NPs with cells grown as a 2D culture and probing the subsequent NP-cell interactions by using optical methods. For DDSNs, fluorescence-based techniques like confocal microscopy and flow cytometry are routinely used to assess NP localisation in the cellular environment and/or the presence of specific extra/intracellular analytes. In vitro imaging can therefore provide insightful information about, for example, changes in intracellular $\mathrm{pH}$ or whether DDSNs functionalised with target moieties specifically recognise extracellular receptors. 
The overexpression of folate receptor allows for cell proliferation through the uptake of folic acid, which promotes DNA synthesis and cell division (Boogerd et al. 2016) and recent findings have shown that folate receptors to be upregulated in several solid tumours. Therefore coating DDSNs with folic acid (folate) has become an attractive route to detect cancerous cells overexpressing folate receptor from non-cancerous cells for theranostic purposes. For example, Wang et al. developed folate-functionalised dyeloaded silica NPs for two-photon imaging and labelling of Hela cells in vitro. These cells over express folate receptor and specifically uptakes the folate-functionalised NPs. NP uptake was reduced when free folic acid was added to the cell culture media and indicated folate-receptor interactions required to enhance uptake. MG63 cells, which have been shown to express low levels for folate receptor (Ross et al. 1994), were also shown to exhibit minimal uptake (Wang et al. 2010). Yang et al. (2011) also used folate-functionalised FITC-loaded silica NPs to target folate receptor-positive KB cells and suggested that NP endocytosis was likely clathrin-mediated. Santra et al. (2005) reported the labelling of human squamous cancer cells (SCC-9) using fluorescein-loaded silica NPs. The use of confocal microscopy then showed that SCC-9 cells were labelled with folate-coated DDNPs and attributed the results to specific folate receptor targeting of the cell surface. However, Ross et al. (1994) have shown that folate receptor expression on SSC-9 cells is in fact low, and highlights how greater experimental evidence proving high receptor expression on cell surfaces is needed to confidently claim targeting is due to specific NP-cell interactions.

Epithelial cell adhesion molecule (EpCAM) is another extracellular receptor that researchers have identified as having a potential role in ontogenesis, and has been targeted by functionalised DDSNs. O'Connell and co-workers (2017) used anti-EpCAMfunctionalised Cy5-doped silica NPs to label and image MCF-7 cells. The $40 \mathrm{~nm}$ NPs were first coated with PEG and then sensitised with anti-EpCAM to facilitate EpCAM targeting on the MCF-7 cell surface. Using confocal microscopy and flow cytometry, the NPs selectively labelled MCF-7 cells and exhibited no specific binding towards EpCAMnegative HeLa cells. MCF-7 cells were considered a model for circulating tumour cells (CTCs), which also overexpress EpCAM. However, the potential of using such DDSNs for labelling, identifying and quantifying CTCs in patient samples is very challenging as there are only few CTCs per billion of blood cells (Shen et al. 2017). The use of antiEpCAM as a targeting biomolecule was also shown by Treerattrakoon et al. (2017) when Cy5-doped silica NPs coated with the monoclonal antibody was used to localise the colloids in HT-29 colorectal tumours.

The adaptable chemistry of silica NPs and possibility to use the nanomaterials as a scaffold have also led to the generation of complex multimodal 'core-satellite' NPs to increase overall NP functionality. For example, Lee et al. (2006) generated dual-mode NPs by attaching iron oxide NPs to the surface of rhodamine-doped silica NPs and enabled MRI and fluorescence imaging of neuroblastoma cells. Cao et al. reported coresatellite use of DDSNs coated with gold NPs for bioimaging. The negatively charged citrate-stabilised gold NPs were electrostatically bound to the positively charged amine-functionalised silica NP surface. The resultant core-satellite NPs were then functionalised with aptamers for the specific recognition and imaging of MCF-7 cancer cells in vitro (Cao et al. 2011). However, while the capacity to form core-satellite 
nanoconstructs was clearly demonstrated, the authors only used the gold NPs as a functional surface upon which to attach thiolated aptamers and cellular imaging was facilitated by the Rubpy-doped silica NPs. Wu et al. (2017) described a thermo-responsive plasmonic core-satellite nanoassembly and employed gold NP satellites anchored to silica NPs via heat sensitive poly( $N$-isopropylacrylamide) for biophotonics and biosensing applications.

Intracellular sensing and imaging has also been reported with DDSNs and can be performed using two different luminescent probes for ratiometric fluorescent sensing; one probe is typically sensitive to analyte while the second is used as a reference (Zou et al. 2016). For example, McDonagh and co-workers used $70 \mathrm{~nm}$ Stöber-synthesised silica NPs for intracellular pH sensing. The NP core was doped with Texas Red (reference dye) while the NP exterior was functionalised with $\mathrm{pH}$-sensitive fluorescein, and demonstrated the capacity for $\mathrm{pH}$ monitoring in human embryonic kidney cell line upon the transition from acidic to basic environment (Korzeniowska et al. 2014).

Intracellular microRNA sensing was demonstrated by $\mathrm{Li}$ and co-workers (2014) in vitro in MCF-7 cells using DDSNs as delivery vehicles for crossing the cellular membrane. The surface of the coumarin-loaded NPs was functionalised with targeting aptamers and a fluorescence-based molecular beacon. The molecular beacon consisted of double stranded DNA: one strand labelled with a fluorescent probe (fluorescein amidite, FAM) and the other was labelled with a quencher (dimethylaminophenylazobenzoic acid, DABCYL). The aptamers allowed for specific recognition of the cancer cells and subsequent NP uptake. Once inside the cells the molecular beacon then preferentially bound miRNA-21, which caused the displacement of the DABCYL-functionalised single strand, and allowed for FAM to fluoresce to indicate the presence of miRNA-21. This strategy was possibly inspired by the use of molecular beacons and gold NPs first described by Mirkin (Seferos et al. 2007).

\section{In vivo and ex vivo}

Fluorescence in vivo imaging typically uses the tissue-penetrable near infrared (NIR) light for image generation. For example, Belfield and co-workers employed folate-functionalised silica NPs for in vivo (one-photon) and ex vivo (two-photon) fluorescence imaging in HeLa xenograft model in mice. A pyran-derivative capable of two-photon absorption and aggregation-enhanced NIR emission was encapsulated into silica NPs and allowed for high loading of the chromophore inside the colloids, with tumour penetration depths of $350 \mu \mathrm{m}$ (Wang et al. 2011b). Biffi et al. developed PEGylated silica NPs $(10 \mathrm{~nm})$ loaded with Cy5.5 and Cy7 in a 2-to-1 ratio for flow cytometry and in vivo imaging applications. In healthy mice, NPs accumulated mostly in the liver and intestine after $24 \mathrm{~h}$, with a negligible amount found in the spleen or brain. After $96 \mathrm{~h}$, a reduction in NP accumulation throughout the mouse was observed, but the largest remaining quantity was found in the liver. No fluorescence could be detected in urine as the NPs were greater than the $8 \mathrm{~nm}$ renal glomerular filters. In lymphoma xenograft tumours, the NPs were detected in tumours $24 \mathrm{~h}$ post-injection and subsequently cleared after $48 \mathrm{~h}$ (Biffi et al. 2014). In spite of these interesting results, the authors did not quantify the percentage of NPs that reached the tumour site and only report 'significant accumulation'. This lack of quantification is widespread in the literature and is also relevant to intravenously 
administered NPs that are intended for cancer treatment. In fact, it has been reported that only around $0.7 \%$ of administered NPs reach tumours (Wilhelm et al. 2016) and highlights the reality that efficacious NP-based imaging and drug delivery agents are extremely challenging to engineer.

Indocyanine green (ICG) is an injectable FDA-approved NIR dye used in the clinic that is considered non-toxic but suffers from a range of issues that has hindered its use in fields like image-guided surgery: poor circulation half-life (Kitai et al. 2005), tendency to bind commonly found serum proteins (Desmettre et al. 2000; Mordon et al. 1998) and low quantum yield in water and blood (Hong et al. 2016). To this end, silica NP formulations have been reported as a way to improve the stability of ICG. One challenge associated with ICG encapsulation inside silica NPs is the inherent negative charge of the dye, which makes it difficult to entrap within the negatively charged particle matrix. Quan et al. addressed this issue by complexing positively charged polyethylene imine with ICG and subsequently doping them into the silica matrix. The ICG-doped silica NPs were then capable of being detected at a 2-cm depth in porcine muscle (Quan et al. 2012).

The biodistribution and clearance of ORMOSILs was performed in healthy, nontumour bearing mice (Kumar et al. 2010). The NIR dye DY776 was covalently incorporated inside the ORMOSIL matrix, with the resultant dye-doped NPs then being functionalised with PEG. The NP surface was subsequently radiolabelled with iodine-124 and the resultant 20-25 nm NPs offered dual-mode in vivo imaging capability. The ORMOSILs largely accumulated in the liver and spleen after $24 \mathrm{~h}$ and greatest fluorescent signal $72 \mathrm{~h}$ post-injection came from the skin, which then gradually decreased until the end of the study where only $3 \%$ of the original fluorescent signal was distributed in the stomach and intestine $(360 \mathrm{~h})$. Interestingly, this skin signal was later adjudged to have come from the DY776 released via NP dissolution. Almost $100 \%$ of the injected ORMOSILs were eventually excreted via hepatobiliary transport, which is not the desired route as the reticuloendothelial system exhibits longer retention compared to renal excretion. Nonetheless, tissue sections of a number of organs indicated no toxicity upon NP exposure and the physical and neurological wellbeing of the mice after 1 month indicated no changes in behaviour or physical features.

The most impactful results for DDSNs in in vivo imaging have been reported by the Wiesner group and their translation to human clinical trials for metastatic melanoma cancer imaging (Ma et al. 2015, 2016; Phillips et al. 2014). The surface chemistry of the Cy5-loaded sub-10 nm NPs employs PEG terminated with ${ }^{124}$ I-labelled cyclic-RGDY peptide (Fig. 6) (Phillips et al. 2014). The arginine-glycine-aspartic acid tripeptide (RGD) is known to bind to $\alpha_{v} \beta_{3}$ integrin receptor (ABIR), which is overexpressed in some cancer cell lines. The NPs therefore have dual imaging capability via Cy5 fluorescence and ${ }^{124}$ I positron emission tomography (PET), and a functionalised PEG layer that allows for integrin targeting via cRGDY peptide. Under Investigational New Drug status from the FDA, initial human studies (Trial number NCT01266096) have shown that the C-dots were well-tolerated by patients, were renally excreted (Fig. 6a, b) and could even accumulate in tumour sites (Fig. 6c-e). However, as the number of patients was limited to 5 , it is thought that a larger participant number is needed to confirm the lack of toxicity and tumour targeting, but the results suggest these C-dots could be a promising cancer imaging agent in the future. Another study aimed for head and 

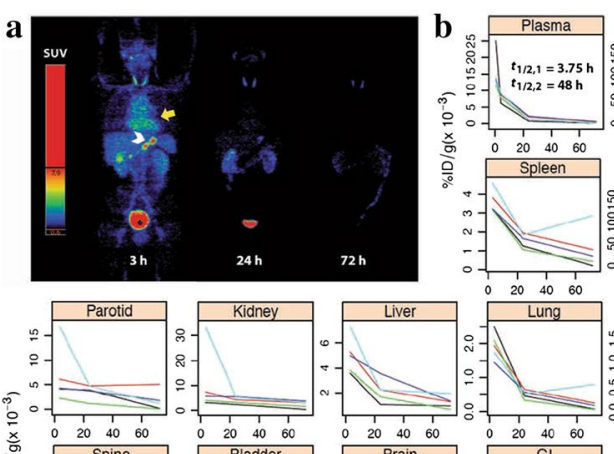

高竞
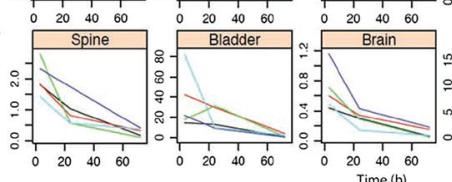
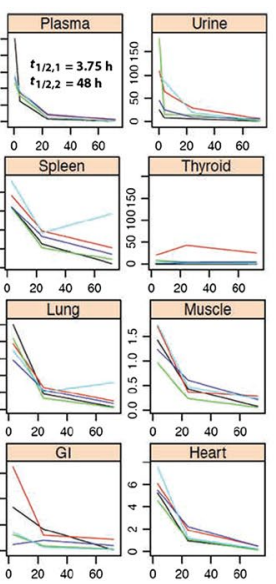

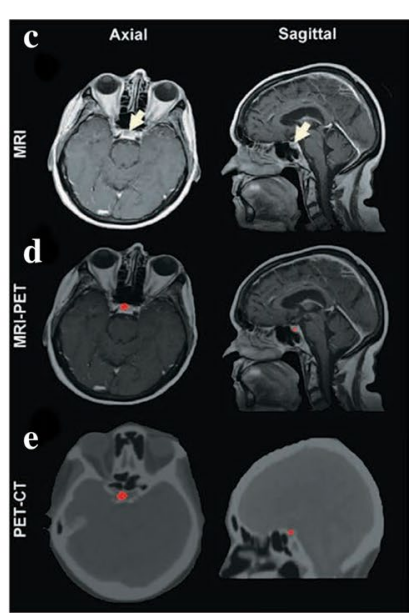

Fig. 6 Bioimaging clinical trials in humans using C-dots. a, b Whole-body distribution and PK of ${ }^{124}$-CRGDYPEG-C dots. a Maximum intensity projection PET images 2, 24, and $72 \mathrm{~h}$ after IV injection of ${ }^{124} \mathrm{I}$-CRGDYPEG-C dots demonstrated probe activity in the bladder $\left({ }^{*}\right)$, heart (yellow arrow), and bowel (white arrowhead). b Decay-corrected percent injected dose per gramme (\%lD/g) of urine and plasma collected around $30 \mathrm{~min}, 4 \mathrm{~h}, 24 \mathrm{~h}$, and $72 \mathrm{~h}$ after injection determined by gamma counting. Regions of interest were drawn on major organs for each patient's PET scans to derive standardised uptake values and $\% \mathrm{ID} / \mathrm{g}(n=5$ patients). Plasma clearance half-times, $t_{1 / 2,1}$ and $t_{1 / 2,2}$ were attributed to renal and hepatobiliary clearance, respectively. c Magnetic resonance, MR, axial and sagittal images of the brain showing a subcentimeter cystic focus (arrows) in the pituitary gland. $\mathbf{d}$ MRI-PET images showing localisation of the C-dots (red spot) in the lesion site. e PET-CT images showing the same localisation (adapted from Phillips et al. (2014))

neck cancer imaging is also underway using these ultrasmall DDSNs (Trial number NCT02106598).

Here, the NPs aim to be used for real-time imaging agents for sentinel lymph node mapping during surgeries, thus potentially allowing surgeons to detected diseased nodes with a hand-held camera. Targeting peptides on the NP surface aim to specifically recognise cancerous tissue such that surgeons can avoid the resection of healthy lymph node. This strategy aims to improve upon current sentinel lymph node mapping approaches using dyes that insufficiently accumulate in nodes and exhibit short retention times (Hameed et al. 2019).

Others have also reported the use of peptide-functionalised DDSNs. For example, Wu et al. (2008b) used RGD-coated fluorescent silica NPs to target and image ABIR-positive MDA-MD-231 breast cancer in vitro and ex vivo. In their mouse xenograft model, the peptide-labelled NPs achieved maximal labelling capacity 1 -h post-injection. However, after $48 \mathrm{~h}$, minimal fluorescence from tumours was observed and suggested the need to improve NP circulation times.

Functionalising silica NPs with the well-known cell penetrating peptide TAT (trans-activating transcriptional activator), which is derived from HIV, has also proven useful as an efficient way of labelling cells and transporting cargo across cell membranes. Santra et al. (2004), for example, demonstrated how $70 \mathrm{~nm}$ FITC-loaded silica NPs conjugated with TAT peptide could successfully be used to image A549 lung carcinoma cells in vitro and brain blood vessels in vivo, with the authors claiming to cross the blood brain barrier without compromising its integrity.

Overall, silica NPs have been widely used for in vitro and in vivo imaging due to the multiple synthetic approaches to doped the material with fluorescent dye but 
also to adapt the particle synthesis to a given requirement. One important consideration in the use of DDSNs in bioimaging is their capacity to be degraded. After exposure to biological fluid silica NPs can be etched and hollowed, consequently causing a release of encapsulated material. While this can be advantageous for controlled drug release applications, for imaging it means released chromophores can cause background noise and erroneous conclusion on the NP intracellular trafficking and biodistribution. While this issue was partially addressed by Mahon et al. (2012b) when they adapted their NP synthesis protocols to increase particle stability in vitro, further studies are needed to test particle stability in vivo.

The ability to control DDSN synthesis is another attractive feature associated with using dye-doped silica in in vivo imaging. This is particularly evident for the case of C-dots being used in human trials where monodisperse sub-10 nm NPs are needed for renal clearance. Ultrasmall Silica NPs (AGuIX NPs, $\sim 5 \mathrm{~nm}$ ) are also being used (as radiosensitisers) in human trials and it is striking that a similarity between both these two silica NP platforms is their ultrasmall size (Lux et al. 2019). Considering both have reached human trial stage, it suggests that small silica NPs may be required for translation from the bench to bedside.

\section{Disease treatment}

\section{Cancer therapy}

Drug delivery applications using silica NPs for the delivery of therapeutic agents to disease sites have largely focussed on cancer treatment and transportation of colloids to tumour sites via the blood. Strategies are typically founded on the idea of exploiting the EPR effect; an effect only known to be observed in cancerous tissue (Peer et al. 2007). Tumours are generally reproduced in vivo using xenograft or orthotopic murine models and therefore allows for NP circulation, tumour accumulation and drug delivery to be studied. However, it should be noted that it can be challenging to realistically replicate human-like tumour environments using these models (Nichols and Bae 2014) but mice studies remain useful for early stage in vivo experimentation.

Traditional chemotherapeutics have been shown to be capable of being encapsulated inside silica NPs and used for cancer treatment. Roy et al., for example, prepared doxorubicin-loaded ORMOSILs of 30, 50 and $80 \mathrm{~nm}$ diameter using vinyltriethoxysilane as a NP precursor via oil-in-water microemulsion. The $50 \mathrm{~nm}$ NPs were shown to exhibit optimal drug release over a 2-week period to pancreatic MiaPaCa-2 cells, whilst unload NPs were deemed non-toxic (Roy et al. 2014).

Gene delivery is another method of cancer therapy. For example, Tivnan and Orr et al. (2012) reported how NIR664-doped silica NPs loaded with microRNA could specifically target and kill neuroblastoma tumours in vivo using a murine xenograft model (Fig. 7). The NP core was loaded with miR-34a, a microRNA that targets genes associated with cell proliferation and apoptosis, and the particle surface was sensitised with an antibody that specifically binds to disialoganglioside, a glycolipid that is expressed in high levels on the surfaces of neuroblastoma cells. The particles also showed minimal uptake in organs and a high degree of biocompatibility. NP dissolution via hydrolysis was proposed as the release mechanism of miRNA from the particles. 


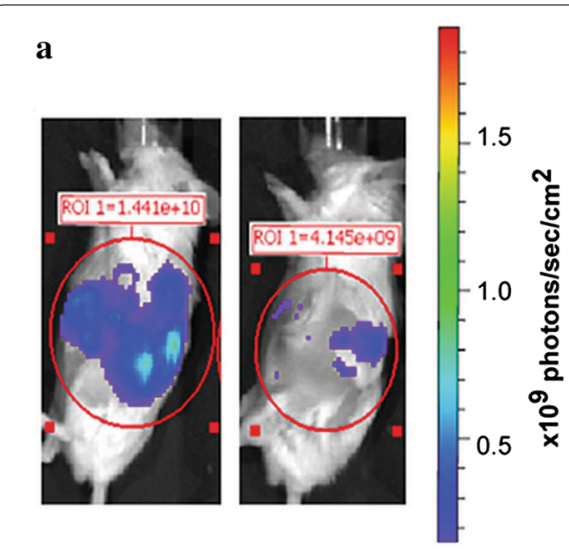

b
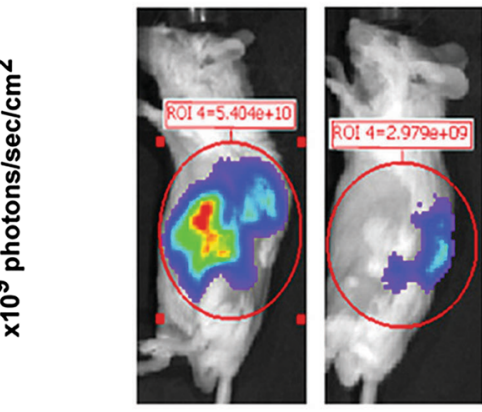

1.5
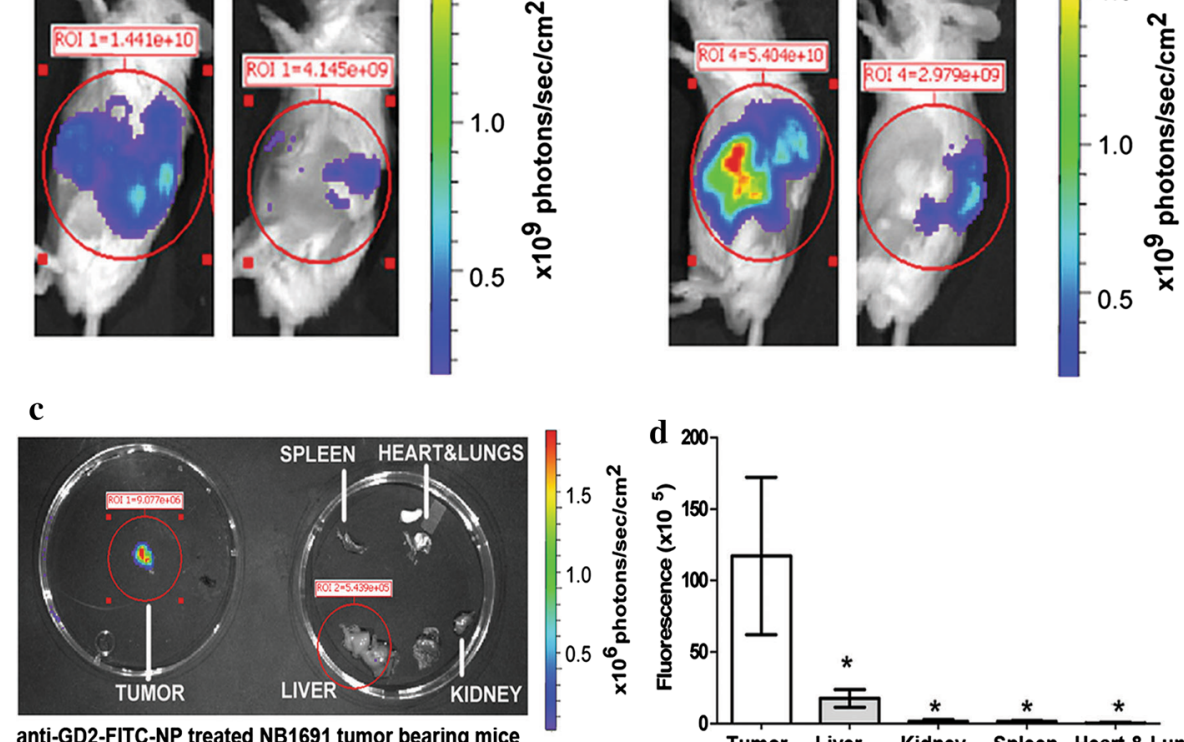

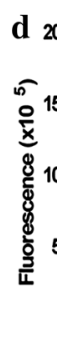

Fig. 7 Delivering therapeutic microRNA with silica nanoparticles for cancer therapy. Anti-disialoganglioside $\left(G_{2}\right)$ sensitised DDSNs were used to treat neuroblastoma in mouse xenograft model. The NPs contained a NIR dye as a contrast reagent and were loaded with therapeutic miRNA34-a. a (left) Antibody-coated NPs containing a scrambled RNA sequence or (right) miRNA34-a were injected into GD2-positive NB1691 tumours and only those containing miRNA34-a reduced tumour size, $\mathbf{b}$ and was also the case for GD2-positive SK-N-AS tumours. c The NPs only tended to accumulate in the tumour tissue and avoided retention in organs like the liver and spleen and $\mathbf{d}$ biodistribution was quantified by fluorescence (adapted from Tivnan et al. (2012))

Ye et al. also demonstrated the ability to deliver genetic material in in vitro and in vivo experiments when they used peptides for synergistic cell penetration and endosomal escape of gelatin-coated silica NPs. The combined presence of TAT peptide and fusogenic peptide HA2 on the NP exterior allowed for the successful transfection of luciferase DNA plasmid pGL3 (Ye et al. 2012). This work was later extended when gelatin-coated silica NPs were functionalised with tumour targeting aptamers and PEG for extended circulation time (Zhao et al. 2016).

The ultrasmall DDSNs developed by the Wiesner group were also studied for their drug delivery capabilities. For example, Yoo et al. reported the design of NP-drug conjugates. The authors reported the synthesis of enzyme-cleavable peptide-drug constructs, and the subsequent conjugation of the constructs to the surface of 6-7 nm silica NPs. The presence of proteases led to the cleavage of the linker-drug constructs, and, in vitro, could reduce phosphorylated epidermal growth factor receptor (EGFR) levels in human tumour-derived H1650 cells (Yoo et al. 2015). The same ultrasmall silica NPs have also been shown to be capable of inducing ferroptosis in nutrient-depleted cancer cells (Dixon et al. 2012).

Ferroptosis is a form of regulated cell-death mechanism induced by specific changes in the intracellular environment, notably severe lipid peroxidation which relies on ROS generation and iron availability (Galluzzi et al. 2018). 
Radiotherapy using NPs is an increasingly studied field in cancer theranostics and gold NPs have featured prominently in research (Jain et al. 2011; McMahon et al. 2011; Hainfeld et al. 2004; Subiel et al. 2016). However, one class of silica NPs known as AGuIX NPs have shown promising results to date and have demonstrated their efficacy in a variety of cancer models [e.g. pancreatic (Detappe et al. 2016), lung (Dufort et al. 2014), bone (Miot-Noirault et al. 2014)]. This has led to a human-based clinical trial (NANO-RAD, Trial number NCT0282045) focusing on the radiosensitisation of brain metastases that originated from lung, melanoma, colon and breast cancers. The AGuIX NPs demonstrated accumulation in brain tumour sites that were previously detected by conventional $\mathrm{T}_{1}$ MRI contrast reagents, as well as a lack of nanomaterial detected in healthy brain tissue. NPs accumulated in tumour sites despite passive targeting and this was attributed to the ability of the nanomaterial to cross the bloodbrain barrier and exploit the EPR effect. Another clinic trial using AGuIX NPs (NanoCOL, Trial number NCT03308604) is also underway and aims to evaluate the human tolerability and safety of increasing doses of AGuIX NPs used in combination with brachytherapy therapy and cisplatin treatment in locally advanced cervical cancers. Similarly to the Cornell dots developed by Weisner et al. (Phillips et al. 2014), the use of ultrasmall NPs is employed as a way of maximising renal clearance of intravenously administered particles that do not localise in tumour sites.

Photodynamic therapy (PDT) and photothermal therapy (PTT) using NPs are forms of physically based treatment gaining increased interest from scientists as a way of overcoming chemotherapy-resistant cancers and typically rely on NIR excitation sources as it can penetrate millimetres into the skin (Henderson and Morries 2015; Ash et al. 2017). PDT uses a photosensitiser which, upon exposure to light, generates singlet oxygen $\left({ }^{1} \mathrm{O}_{2}\right)$ and acts as a reactive species that can ultimately cause cell death. Benachour et al. used gadolinium-loaded silica NPs and a chlorin photosensitiser to facilitate dual-functional MRI and PDT. The NPs were functionalised with peptides that allowed for retention of the NPs in intracranial tumour sites in a rat xenograft model, thereby facilitating brain imaging (Benachour et al. 2012). Ohulchanskyy et al. (2007) modified an iodine-exhibiting pyropheophorbide derivative to allow for the photosensitiser to be covalently linked inside the silica matrix and then used the colloids to kill Colon-26 cells. In another study, an aggregated fluorescent dye capable of two-photon excitation was encapsulated inside the matrix of the $30 \mathrm{~nm}$ ORMOSIL NPs and used as a FRET donor for sensitising a co-encapsulated 2-devinyl-2-(1-hexyloxyethyl)pyropheophorbide, HPPH. Indirectly exciting HPPH via energy transfer from the aggregated dyes generated singlet oxygen intracellularly and led to cancer cell toxicity in vitro (Kim et al. 2007).

Singlet oxygen generation using photosensitisers has also been shown to be more efficient in the presence of heavy atoms, an effect known as the heavy atom effect. Here the photophysical and biological properties of photosensitisers can be optimised by incorporation functional group containing heavy atom such as bromine (Gorman et al. 2004). The number and the localisation of bromine substituents can tune the efficiency of the electronic transition from a singlet to a triplet state, leading to more effective energy transfer from the excited derivative onto oxygen. Zheng exploited this by synthesising a silylated boron-dipyrromethene (BODIPY) derivative containing iodine atoms and subsequently covalently bound this probe into silica NPs (Wang et al. 2015). The NPs 
were incubated with HeLa, MCF-7 and SKBR-3 cells and showed significant cytotoxicity upon laser exposure compared to NPs doped with BOPIDY without iodine. The authors also suggested replacing the heavy metal with its radioactive form to allow for future PET imaging.

PTT utilises photosensitisers that convert the energy of incident photons to heat. Hyperthermia, the heating of tissue to $41-47{ }^{\circ} \mathrm{C}$ over tens of minutes (Hayashi et al. 2014), causes irreversible cell membrane damage and protein denaturation that eventually leads to cell death. This is particularly useful in hypoxic tissues as oxygen is not required. Shan et al. (2013) showed the PTT capacity of NIR CyTE-777 coated silica NPs to be used as a PTT agent. In vitro experiments demonstrated a $42 \%$ reduction in macrophage viability under $780 \mathrm{~nm}$ excitation. However, the authors stated that their nanocomposite exhibited lower heating efficiency compared to gold nanorods typically used in PTT.

Synergistic dual PTT and PDT has also been reported using $42 \mathrm{~nm}$ porphyrin-loaded silica NPs in vivo (Hayashi et al. 2014). The crosslinking of iodopropyltrimethoxysilane in the NP matrix exploited the heavy atom effect to promote more efficient generation of ${ }^{1} \mathrm{O}_{2}$ and heat. Importantly, the study deviated from laser excitation sources and utilised LEDs for photosensitiser illumination, which reduces the degradation of the sensitiser over extended excitation periods and is more practical for clinical settings.

\section{Non-cancerous applications}

The blood-brain barrier (BBB) is a highly cooperative system composed of endothelial cells, extracellular matrix, pericytes, neurons, astrocytes and a variety of enzymes, receptors, transporters and efflux pumps (Barbu et al. 2009). It is therefore highly challenging to deliver therapeutic agents to the brain and different physical and chemical strategies for BBB disruption have emerged (Zhang et al. 2016). However, these strategies are not without risk as BBB disruption can be can linked to strokes (Sandoval and Witt 2008) and central nervous system diseases (Sweeney et al. 2018). To this end, improved strategies based on NP systems that maintain maximal barrier integrity are required, and because silica is described as a promising drug delivery platform there have been reports of BBB studies with the dye-loaded nanomaterials.

A systematic study by Song et al. (2017) using Ru(bpy)-doped silica NPs produced via the reverse microemulsion method were used to investigate particle transcytosis in vitro BBB model. The NPs $(25 \mathrm{~nm}, 50 \mathrm{~nm}, 100 \mathrm{~nm})$ were PEGylated and further functionalised with lactoferrin, a cationic iron-binding glycoprotein that can bind to the lactoferrin receptor present on vascular endothelial cells of the BBB. Without lactoferrin on the NP surface less than $5 \%$ of particle crossed the BBB, whereas NPs exhibiting the glycoprotein were transcytosed in a size-dependent manner. Reducing NP diameter led to increasing transport efficiency with $25 \mathrm{~nm}, 50 \mathrm{~nm}, 100 \mathrm{~nm}$ quantified to have $21.3 \%, 11.6 \%$ and $5.8 \%$ efficiency, respectively. Importantly the authors also showed that the introduction of the NPs into the BBB system did not affect tight junction integrity as low sodium fluorescein permeability coefficients were calculated. ORMOSILs were also shown to be capable of entering neuronal regions when they were fed to Drosophila (Barandeh et al. 2012). No effect on organism viability was observed despite the NPs penetrating into the brain, neuronal cell body and axonal projections. 
Ku et al. (2010) reported the transcytosis of 80-90 nm PEGylated silica NPs across the tightly bound endothelial cells lining the barrier after $1 \mathrm{~h}$ in rats, whereas non-PEGylated counterparts exhibited no crossing. Transmission electron microscopy (TEM) was used to visually investigate brain slices to elucidate their localisation. Particles were found in astrocytes and it was also confirmed by confocal microscopy that NPs managed to locate in neurons. On the other hand, multiple in vivo studies have shown that NPs are not able of crossing the $\mathrm{BBB}$ (He et al. 2008) and is fact one of the greatest challenges facing the drug delivery community. Discrepancies in experimental outcome can be due to different experimental parameters, with a major contributor being the choice of BBB model. Models exist for different organisms (fruit flies, zebrafish rodents and humans) and differences exist across species. For example, pericytes and astrocytic end feet are more prevalent in the human neurovascular unit compared to mouse brains (O'Brown et al. 2018). BBB in vitro and in vivo models also differ (Stanimirovic et al. 2014). Researcher should therefore consider the model chosen and, in addition, the physicochemical characterisation of NPs in biological fluids when analysing BBB studies.

Silica NPs have shown they can also be useful for treating other diseases such as osteoporosis. Researchers at Emory University have shown that silica NPs are not totally inert and can intrinsically benefit the biological activity of osteoblasts and osteoclasts (Beck et al. 2012; Ha et al. 2014; Weitzmann et al. 2015). Silica NPs themselves stimulate bone-forming osteoblasts, suppress bone-resorbing osteoclasts and enhance bone mineral density in vivo (Beck et al. 2012). Silica NPs therefore could potentially be a novel method of treating osteoporosis and advantageous compared to current treatments that only target osteoclasts but do not rebuild bone mass (Ha et al. 2014; Weitzmann et al. 2015). Following caveolae-mediated endocytosis of the NPs in osteoblasts, a signal transduction pathway dependent on ERK1/2 was stimulated. Autophagic proteins bound to the NPs were processed when ERK1/2 was stimulated and, following processing, led to autophagosome formation (Ha et al. 2014). This cascade of events was common for both $-\mathrm{OH}$ and PEGylated silica NPs and subsequently led to the activation of autophagy, which has been shown to be associated with osteoblast differentiation and mineralisation (Liu et al. 2013).

Silica NPs can also be used to facilitate core-shell architectures, where the shell is composed of a second nanomaterial (e.g. silica-gold). The plasmonic properties of gold can then be used for thermal-based treatments of diseases. For example, Sebashells (produced by Sebacia) have undergone trials for the treatment of dermatological conditions like acne (Fig. 8) (Anselmo and Mitragotri 2015). A thin gold shell around a silica core (Fig. 8a) was used to thermally ablate sebaceous glands and the eradication of the acne source. The NP formulation is applied topically and an ultrasound source encourages the penetration of the NPs deep into the gland (Fig. 8b). NIR light (similar to that used for laser hair removal) is used for tissue penetration and is absorbed by the gold shell. The gold shell converts the absorbed light into heat energy and results in glandular thermolysis (Fig. 8c). The ultrasound and NIR technologies are similar to tools already approved by the FDA and overall the Sebashell treatments appear to have been well-tolerated by patients, but further studies will be required to assess NP clearance (Paithankar et al. 2015). 

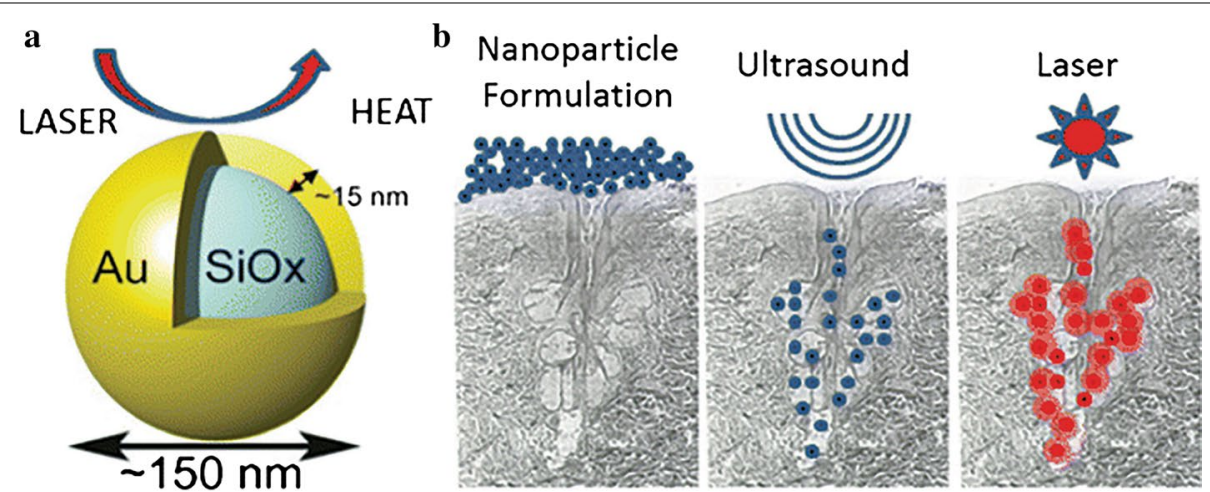

Fig. 8 Silica nanoparticles are regularly used as core materials in core@shell nanomaterials designed for biomedical applications. For example, Sebashells are currently undergoing clinical trials for the treatment of acne. a Particle architecture consists of a silica core and a gold shell. When excited with near infrared light the gold shell heats and ablates sebaceous glands. b Sebashells are applied topically and penetrate sebaceous glands through ultrasound stimulation. NIR laser light is then used for gold shell to induce heat production. $\mathbf{c}$ Such heat treatment can result in deep glandular thermolysis (adapted from Anselmo and Mitragotri (2015))

Overall, it is positive to see silica NPs being used in human trials for cancer treatment (Lux et al. 2019) and represents a breakthrough in the use of silica NPs in disease therapy. It is interesting that the focus of researchers has been to develop ultrasmall NPs that can be cleared through the kidneys as this strategy has been mirrored by the C-dots (Phillips et al. 2014) being used in human bioimaging trials. It is therefore clear that silica NPs have the potential to become reliable and clinically relevant imaging and therapeutic agents. While these clinical trials have primarily studied the safety and pharmacokinetics of their nanomaterials over short time scales (days), the nanomaterials appeared to be well-tolerated. The scientific community should therefore follow the progression of these trials closely and attempt to establish parallels with these technologies that have so far be translatable to humans.

\section{Challenges}

The use of NPs in biological applications is continuously growing in popularity, and it is therefore important to assess NP interactions with complex biological media to better understand the formation of biomolecular coatings on particle surfaces and the resultant biological fate of such particles. A deeper knowledge of these interactions will ultimately lead to better predict toxicity across a range of exposure routes, e.g. upon inhalation, ingestion, injection or via transdermal penetration (Kim et al. 2015). However, the lack of standardisation in nanotoxicology experimentation has led to of conflicting reports on colloidal silica NP biocompatibility in the literature (as is the case for almost all nanomaterials), and has hence evaluating and summarising the effects NPs have on various cells lines and whole organisms is challenging (Gubala et al. 2018a). In addition, it is also crucial to consider that there are multiple forms of nano-form silica employed in bioapplications and that improved physicochemical characterisation of the NPs is required prior to toxicological studies (Gubala et al. 2018b). For example, factors like the choice of synthetic route and crystal structure may affect biological responses (Murugadoss et al. 2017). Researchers must therefore consider silica NPs as a class of nanomaterial with a 


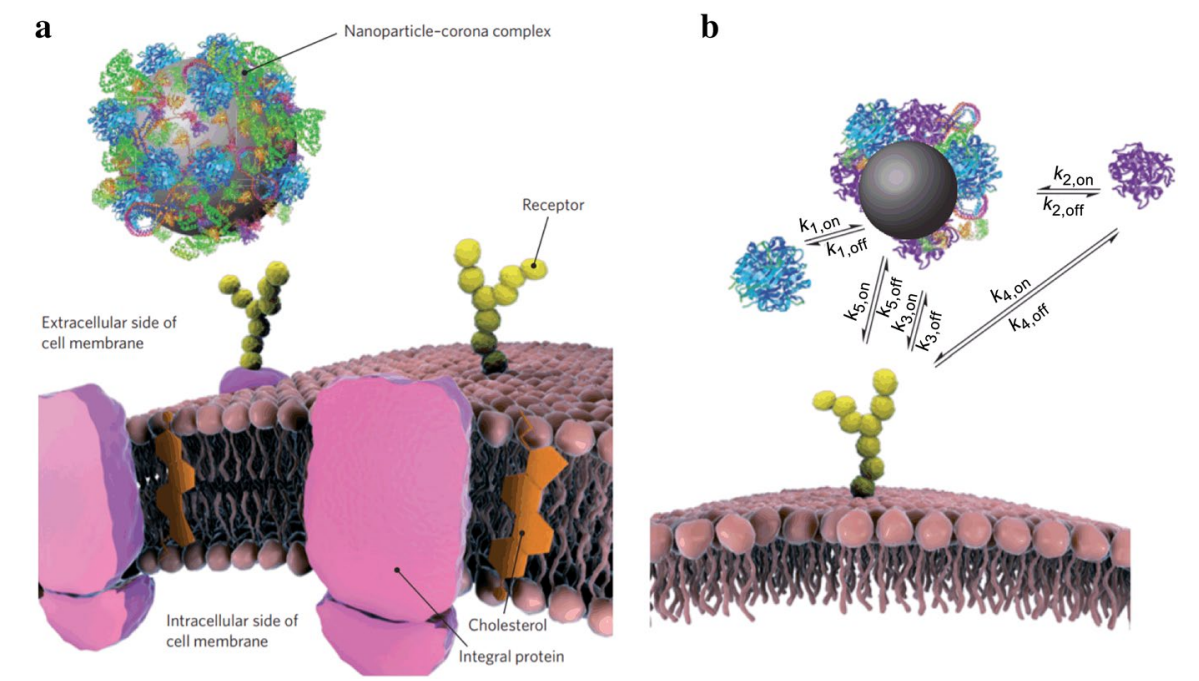

Fig. 9 The protein corona, and its manipulation, poses many new challenges and opportunities in bionanotechnology. a The strong adsorption of biomolecules to a NP surface in complex media (known as the 'protein corona' can alter the biological identity of NPs and has recently been described what a cell 'sees' upon encountering the particle-corona complex. $\mathbf{b}$ A number of association/dissociation reactions occur at the NP-cell interface. Some biomolecules bind tightly to form a 'hard' corona and others loosely surround the NP (a 'soft' corona) (adapted Monopoli et al. (2012))

number of subtypes (e.g. microporous, mesoporous, food grade), each of which should be considered individually and thoroughly physicochemically characterised before biological testing.

\section{Protein corona}

In spite of the efforts made by materials scientists to control and impart specific surface properties to silica NPs, it is now widely accepted that once these nanomaterials are introduced into complex biological fluids like serum, blood or urine the surface properties of the NP can be dramatically changed. This surface fouling is attributed to biomolecule (mainly protein) adsorption around NP surfaces and is referred to as the 'protein corona.' There are two layers of this corona that coat NPs; 'hard' and 'soft' (Fig. 9) (Monopoli et al. 2012). A hard corona is a near-monolayer of biomolecules tightly bound, but not irreversibly, to the NP surface. Surrounding this layer is the 'soft' corona, which is composed of more loosely associated and rapidly exchanging biomolecules. It is the hard corona that is believed to provide the environmentally derived identity of a given nanomaterial that a cell would actually interact with Li et al. (2010), Mahon et al. (2012a). Tenxer et al. (2013) showed that it can take as little as $30 \mathrm{~s}$ for a protein corona to form on silica NPs, and researchers should begin to regard the corona as defining 'the bio-nano interface' that can cause unpredicted interactions with biological systems (Walczyk et al. 2010; Soddu et al. 2019).

For example, Lara et al. (2017) showed that the epitopes of two major proteins in serum, low-density lipoprotein and immunoglobulin G, are presented on the surface of silica NPs and can be recognised by their respective receptors. The authors suggested 
that cells may therefore be 'mistakenly' recognising NPs (based on their corona composition) as endogenous or exogenous objects, such as lipoproteins or viral infections, respectively, and may in turn govern how the NPs are biologically processed and/or cleared.

In the case of nanomedicine this presents a challenge as biomolecule-functionalised silica NPs are designed for recognising specific cellular receptors can lose their targeting capability. In a notable study by Salvati et al. (2013) transferrin-coated silica NPs were shown to lose their ability to target transferring-receptors on cancer cells in vitro. The loss of targeting capability was observed when the experiments were performed in cell culture medium supplemented with serum. Without serum supplementation, no corona was formed and transferrin receptor could be recognised by the transferrin-coated NPs. Kelly et al. (2015) extended this work by visually mapping the reduction of antibodycoated NP functionality upon corona formation using TEM, differential centrifugation and other techniques. Researchers must therefore carefully consider the supposed benefits associated with producing biomolecule-functionalised NPs capable of target recognition in vivo and must interpret experimental results with the side effects of the protein corona in mind. It has also been shown for silica NPs that the physicochemical properties and biomolecular composition of the corona significantly evolves when protein concentrations change from those used in in vitro studies to those found in vivo, and holds deep implications in studying biodistribution, toxicity and a host of biological responses to silica NPs (Monopoli et al. 2011).

It has been hypothesised that precise corona modifications could lead to a greater understanding of how the different biomolecules coating the NPs influence cellular interactions. For example, around $50 \%$ of all human proteins contain glycan units (Bohne-Lang and von der Lieth 2005) that can influence protein immunogenicity, trafficking, stability and folding (Paszek et al. 2014; Moremen et al. 2012) and Wan et al. (2015) demonstrated how their presence/absence in the protein coronas can modulate macrophage interactions. Compared to fully glycosylated coronas, the removal of the glycans led to enhanced NP adhesion and uptake in THP-1 macrophages, which ultimately led to an increase in pro-inflammatory cytokines by the cells.

Another study by Monopoli and co-workers developed an analytical method for modifying a human plasma-derived protein corona on silica NPs through the use of surfactants. Cholamidopropyldimethylammonio propanesulfonate (CHAPS), a zwitterionic surfactant, could selectively extract apolipoprotein AI (ApoAI) from the hard corona formed on SiNPs without affecting particle size. Silica NPs with ApoAI-depleted coronas did not aggregate upon the introduction of anti-ApoAI and suggested the possibility of engineering the hard corona to reduce specific biological interactions. On the other hand, the introduction of anti-ApoAI caused silica NPs untreated with CHAPS to aggregate in the presence of ApoAI and suggested the presentation of binding epitopes in the outer layer of the hard corona. The authors also commented that removing specific proteins would subsequently result in new protein binding sites to be exposed on the corona surface and would possibly lead to different interactions of the NP with cellular machineries (Maiolo et al. 2014).

Further studies are needed to fully understand the influence of corona in receptor activation, signal transduction and biodistribution of NPs, but it is encouraging to see how 
the scientific community is attempting to answer fundamental questions posed by the use of NPs in biological environments. Improvements in the ability to manipulate the corona will undoubtedly lead to greater understanding of NP interactions with cellular machinery, and it has been predicted that it may eventually be possible to produce more favourable cell targeting with NPs to result in more efficacious disease treatment (Ke et al. 2017; Mahon et al. 2012a).

Despite the numerous research papers in the field, it is clear that multiple parameters affect the biomolecular corona formation, such the temperature of the incubation, the time, the total mass of the biomolecules in the exposing media type, along with the analytical techniques to isolate and to characterise the corona complexes. Such techniques either directly or indirectly measure corona formed on NPs and the use of complementary analytical methods is advised (Carrillo-Carrion et al. 2017). A recently publication has highlighted the characterisation challenges associated with protein corona investigations and a minimal information about nanomaterial-biocorona experiments has been suggested (Chetwynd et al. 2019).

The importance and relevance of the corona has begun to attract attention in several regulatory documents, although its characterisation is not a requirement yet. In particular, the European Food Safety Authority (Hardy et al. 2018), International Life Sciences Institute (Cockburn et al. 2012) and the US Food and Drug Administration (US FDA Center for Drug Evaluation and Research 2017) have all highlighted that the corona is a factor that has to be taken into consideration in the evaluation of the nanomaterials although the community is still investigating the minimal parameters that are required. The Scientific Committee on Emerging and Newly Identified Health Risks of the European Commission has also noted, in an opinion on the determination of potential health effects of nanomaterials used in medical devices (2015) that "because nanomaterials may acquire a new biological identities, i.e. new properties via the adsorption of biomolecules (the bio-corona) onto their surface, it is essential during toxicological studies to assess the interaction between these and how these may interact with the physiological response of the organism" (European Commission 2015). Overall, it is clear that assessment of the bio-corona on NBMs is important, yet this currently represents a regulatory gap in nanomedicine the importance of the bio-corona as a factor that has to be taken into consideration in the evaluation of nanomaterials (Halamoda-Kenzaoui et al. 2019).

\section{Toxicity}

Food grade synthetic amorphous silica (SAS) is a food additive (E551, silicium dioxide) and is used as an anti-caking agent in food products. An informative overview of SAS safety by Fruijtier-Pölloth (2016) concluded that, based on current evidence obtained by various global regulatory bodies, no significant risk to human health is posed by E551. However, SAS is considered to be a different class of material to the colloidal suspensions synthesised by methods outlined earlier in this review. Therefore, SiNP would not comply with the specifications established for E551, and would require their own application-specific safety and risk assessments. It is therefore crucial to note that colloidal silica will not necessarily be non-toxic and safe to use in vivo simply because SAS has so far been deemed to be of low risk to human health (Fruijtier-Pölloth 2016). In this light, the human clinical trials using DDSNs from the Wiesner group for bioimaging 
(Phillips et al. 2014; Benezra et al. 2011) will provide important insights into silica NP tolerance and should enable a number of new paradigms for this class of nanomaterial to be established.

Some reports have described silicon-based materials as naturally biodegradable and that they dissolve into silicic acid (a naturally occurring compound) (Park et al. 2009; Kempen et al. 2015). However, assessing the toxicity of colloidal silica NPs remains unresolved and is particularly hampered by the lack of agreed experimental protocols. This has meant a variety of different experimental parameters have been studied: NP size (Kim et al. 2015) and agglomeration-state (Giovannini et al. 2018b; Halamoda-Kenzaoui et al. 2017), NP surface chemistry (Hsiao et al. 2019), NP corona composition, (Lee et al. 2015) particle concentration (Kim et al. 2015) and cell type (Kim et al. 2015). This wide variation in experimental conditions has therefore led to a lack of consensus on whether colloidal silica can be definitively considered to be toxic (Krug 2014).

The presence of serum in cell culture media used for in vitro studies is now regarded as a key experimental parameter as it can influence cellular responses to NP exposure (Francia et al. 2019). Scrutiny of experimental conditions is therefore important and, in the case of in vitro experiments conducted in the absence of serum, caution should be taken when interpreting NP-cell interactions. For example, viability studies in mouse keratinocytes using silica NPs with diameter 30, 48, 118 and $535 \mathrm{~nm}$ showed decreased cell survival with decreasing size across a dosage range of 10-200 $\mu \mathrm{g} / \mathrm{mL}$ (Yu et al. 2009). However, NPs were exposure to cells in culture media in the absence of serum and results/trends should be interpreted in this context. A different study involving human hepatocytes employed silica NPs with diameters of 7, 20 and $50 \mathrm{~nm}$ at dosages of $20-640 \mu \mathrm{g} / \mathrm{mL}$. Cells were cultured in the presence of serum and viability reduced in the order of $20>7>50 \mathrm{~nm}$ (i.e. no clear size dependence), and was dose and exposure-time dependent ( $\mathrm{Lu}$ et al. 2011). The ability to establish toxicological trends, like an overall size-dependent toxicity, is therefore hampered by such inconsistencies in experimental procedures.

Kim and co-workers (2015) evaluated cytotoxicity of different concentrations of 20, 60, 100 and $200 \mathrm{~nm}$ silica NPs produced by Stöber method in A549 (lung), HepG2 (liver) and NIH/3T3 (fibroblasts in blood circulation). It was noted that NP toxicity was size-, concentration- and cell line-dependent and noted that $60 \mathrm{~nm}$ particles were preferentially endocytosed by cells and high doses induced a disproportionately large decrease in cell viability. While the study was thorough and accounted for a number of toxicity endpoints (e.g. ROS, cell membrane integrity), the zeta potential was only measured in DI water and varied between -24 and $-38 \mathrm{mV}$ for the 4 sizes of NP, but would have been further improved by measuring in more biologically relevant media.

Zielinski et al. explored potential side effects of using their silica NP-embedded biodegradable scaffolds intended for laser-tissue-soldering of blood vessels in the brain. It was presumed that upon scaffold degradation the silica NPs would be released into surrounding brain tissue and uptaken by cells, thus possibly causing unintended neurotoxicity. The negatively charged NPs were coated with either ICG/poly ( $\varepsilon$-caprolactone) or ICG/poly ( $\varepsilon$-caprolactone-poly L-lactide) and oxidative stress was induced in microglial cells, thereby potentially contributing to neuronal injury (Koch et al. 2014). Further investigations highlighted that the same NPs were 
predominantly uptaken via macropinocytosis and phagocytosis and were detected in the lysosomes and the endoplasmic reticulum after $4 \mathrm{~h}$ exposure (Zielinskia et al. 2016). Another study investigating in vitro neurodegeneration demonstrated that ICG/poly ( $\varepsilon$-caprolactone)-coated silica NPs affected mitochondrial function in model SH-SY5Y cells during differentiation, with the authors concluding that further studies are required to understand the potential for silica NP-induced neurodegeneration (Ducray et al. 2017).

Alternative approaches to assess longer-term effects of NP exposure are also needed to achieve a better understanding of the potential risks associated with Si NP exposure. For example, deviating from cytotoxicity experimentation (which typically only last a number of days) and understanding the genetic responses of organisms to NP exposure could also be highly useful. This should allow researchers to form a more comprehensive perspective on the interaction between nanomaterials and biological systems on a longer-term basis. For example, Hu et al. (2016) performed genome-wide analysis to uncover the genetic pathways associated with the toxicity their silica NPs induce in zebrafish embryos. The cytotoxic effects of the NPs resulted in the alteration of gene expression patterns associated with various biological processes, including immune response, response to stimuli and embryonic development. Overall, the expression of 2515 genes were altered, with 1107 being up-regulated and 1408 down-regulated. Similar studies need to be more widely performed such that a broader insight into the biological impacts of nanomaterials can be obtained.

The majority of toxicity studies also usually ignore how the protein corona influences NP-cell interactions despite strong evidence suggesting that it can (Corbo et al. 2016). For example, an interesting study by Wang et al. (2017) demonstrated how $100 \mathrm{~nm}$ silica NPs, when exposed to lung tissue, specifically bind transforming growth factor $\beta 1$ (TGF$\beta 1$ ) in their protein corona and ultimately induces the development of lung fibrosis in murine models. The high concentration of TGF- $\beta 1$ in the silica NP corona prolonged activation of the TGF- $\beta /$ Smad2 pathway, which promotes tissues fibrosis. Specifically, the authors suggest that occupational lung diseases like silicosis could potentially be initiated by the corona composition, and demonstrate how the corona formation on NPs can adversely induce pathological changes in biological systems. In fact, to further complicate matters, a report has shown that the common methods used to isolate NPcorona complexes (prior to removal of the proteins from the silica NP surface) can influence the abundance of certain proteins in the corona and also its rearrangement (Pisani et al. 2017).

In addition, major areas toxicology are largely unexplored. For example, it is known that the microbiota plays a crucial role in human health and that it's dysbiosis can be linked to different forms of disease (Mohajeri et al. 2018). The risks and potential opportunities associated with NP-microbiome interactions have only become more heavily researched in the recent years but it is clear from studies that forms of dysbiosis occur upon encountering nanomaterials (Poh et al. 2018; Bouwmeester et al. 2018). However, It is not always clear whether nanoparticles directly cause dysbiosis or whether the effects are attributed to their dissolved/molecular form (caused, for example, by nanomaterial dissolution), or a combination of both. Considering humans encounter nanoparticulate matter over the course of their lives (e.g. in food, cosmetic products), it is likely that 
microbiota studies will become a core focus of human toxicology in the future. Coupled with our increased understanding of the protein corona and its ability to influence bionano interactions, the challenges facing the scientific community to develop safe nanomaterials continue to be non-trivial. More fundamental research into uncovering the intricacies of bio-nano interactions is required, and is particularly necessary since the material scientists continue to develop increasingly more sophisticated nanomaterials for bioapplication before fully considering the associated future risks. To this end, it is encouraging to see that regulators are increasingly aware of the hazards posed by nanomaterials intended for human exposure (Halamoda-Kenzaoui et al. 2019).

\section{Conclusion}

This review has aimed to summarise important literature associated with silica NP synthesis, functionalisation, bioapplications and the importance of the protein corona. The two common synthetic approaches to producing silica NPs, namely Stöber method and reverse microemulsion, were described. Considerations associated with silica NPs doping with fluorophores were also highlighted and different bioconjugation strategies used for the creation of biomolecule-coated silica NPs were discussed.

It is clear from the literature that DDSNPs are finding success in biomedical diagnostic applications. For fluorescence-based tests, the ability of DDSNs to outperform traditional organic fluorophores as reporters has led to their incorporation into pointof-care devices. DDSNs have also become more commonly employed in bioimaging due to the ability to be designed to suit different biological applications that require adaptable optical platforms. One particularly exciting development in the application of DDSNs has been the emergence of C-dots as promising in vivo optical probes for tumour imaging. Additionally, their tendency to accumulate in tumours may mean they have the potential to deliver drugs to tumour beds. Indeed, it is also evident that success has been found when applying DDNPs to in vivo drug delivery applications, most notably AGuIX NPs. However, reports published in mouse models have shown promising trends like good biodistribution profiles and rapid tumour reduction, but very few DDNPs have been used in human studies (as has been the case for practically all NP types). For this reason further results of C-dot bioimaging clinical trials in humans are much anticipated.

Most strategies for localising NPs in tumour sites are based on the exploitation of the EPR effect, a method used in clinically successful liposome formulations like Doxil. However, in spite of intensive efforts, very few NP-based imaging or therapeutic agents have been translated to the clinic. This may be due, in part, to the inaccurate in vitro and in vivo models that do not fully replicate human tumoural environments (e.g. chemical environment, architecture complexity and interactions with other healthy tissues). The more widespread use of improved cancer models is therefore needed to ensure more scientists are testing NPs in 'human-like' conditions (Katt et al. 2016). It is here that further understanding the role played by the protein corona on the overall fate of NP in vivo will undoubtedly be necessary when attempting to predict the NP fate. Additionally, the evaluation of the potential longer-term effects of NP interactions with biological systems remains understudied and is an area of nano-risk assessment that requires improvement. For example, improved in vitro 
studies where large scale testing can be performed using human-like conditions will prove to be beneficial [e.g. using organoids (Truskey 2018)]. Techniques like grouping and read across may also prove useful in avoiding case-by-case studies and streamlining nanorisk assessments (Oomen et al. 2018; Mech et al. 2019). If progressions are made in such areas then the outlook that DDNPs will deliver on their promise and contribute to the predicted nano-revolution in healthcare is positive, and it is therefore expected that DDSNs will continue to be widely used in bio-nanotechnology.

\begin{abstract}
Abbreviations
ABIR: $\alpha_{v} \beta_{3}$ integrin receptor; AFP: a-fetoprotein; APTES: aminopropyltriethoxysilane; APTMS: aminopropyltrimethoxysilane; ApoAl: apolipoprotein Al; BBB: blood brain barrier; BODIPY: boron-dipyrromethene; $C$ dot: Cornell dot; $C^{\prime}$ dot: Cornell prime dot; CHAPS: cholamidopropyldimethylammonio propanesulfonate; CTC: circulating tumour cell; DABCYL: dimethylaminophenylazobenzoic acid; DDSN: dye-doped silica NP; EDC: ethyl(dimethylaminopropyl) carbodiimide; EGFR: epidermal growth factor receptor; ELISA: enzyme-linked immunosorbent assay; EPCAM: epithelial cell adhesion molecule; EPR: enhanced permeability and retention; FAM: fluorescein amidite; FITC: fluorescein isothiocyanate; FLISA: fluorescence-linked immunosorbent immunoassay; FRET: fluorescence (or Förster) resonance energy transfer; HPPH: 2-devinyl-2-(1-hexyloxyethyl)pyropheophorbide; HRP: horseradish peroxidase; ICG: indocyanine green; IgM: immunoglobulin M; II-6: interleukin-6; MiRNA: microRNA; NHS: N-hydroxysuccinimide; NIR: near infrared; NMR: nuclear magnetic resonance; NP: nanoparticle; ORMOSIL: organically modified silica; PAMAM: polyamidoamine; PDT: photodynamic therapy; PEG: polyethylene glycol; PET: positron emission tomography; PLuS NPs: Pluronic silica NPs; PPO: polypropylene oxide; PTT: photothermal therapy; PVP: polyvinylpyrrolidone; ROS: reactive oxygen species; RGD: arginine-glycine-aspartic acid; $\left[\text { Ru(bpy) }{ }_{3}\right]^{2+}$ : tris(bipyridine) ruthenium(II) chloride; SAS: synthetic amorphous silica; STED: stimulated emission depletion; TAT: trans-activating transcriptional activator; TEOS: tetraethyl orthosilicate; TEM: transmission electron microscopy; TGF- $\beta 1$ : transforming growth factor $\beta 1$; TMOS: tetramethyl orthosilicate.
\end{abstract}

\title{
Acknowledgements
}

The authors gratefully thank Dr. Delyan R. Hristov and Mr. Antonio Aranda-Ramos for feedback and discussion during the preparation of this manuscript.

\section{Authors' contributions}

CJM provided substantial contribution to gathering literature, to intellectual input and to writing the manuscript. VG, GG, FK, MPM gathered relevant literature, provided intellectual input and contributed to the writing of the manuscript. All authors read and approved the final manuscript.

\section{Funding}

Not applicable.

\section{Availability of data and materials}

Not applicable.

Ethics approval and consent to participate

Not applicable.

\section{Consent for publication}

Not applicable.

\section{Competing interests}

The authors declare that they have no competing interests.

\section{Author details}

${ }^{1}$ Medway School of Pharmacy, University of Kent, Chatham Maritime, Kent ME4 4TB, UK. ${ }^{2}$ EMPA Material Science and Technology, 9014 St Gallen, Switzerland. ${ }^{3}$ National Research Council of Canada, Ottawa, ON K1A OR6, Canada. ${ }^{4}$ Department of Chemistry, Royal College of Surgeons in Ireland (RCSI), 123 St Stephen Green, Dublin 2, Ireland. ${ }^{5}$ Istituto Italiano Tecnologia, 30 Via Morego, 16163 Genoa, Italy.

Received: 30 May 2019 Accepted: 10 December 2019

Published online: 09 January 2020

\section{References}

Ab Rahman I, Padavettan V. Synthesis of silica nanoparticles by sol-gel: size-dependent properties, surface modification, and applications in silica-polymer nanocomposites_a review. J Nanomater. 2012. https://doi. org/10.1155/2012/132424.

Adnan N, Cheng Y, Ong N, et al. Effect of gold nanoparticle shapes for phototherapy and drug delivery. Polym Chem. 2016;7:2888-903. https://doi.org/10.1039/c6py00465b. 
Adumeau L, Genevois C, Roudier L, et al. Impact of surface grafting density of PEG macromolecules on dually fluorescent silica nanoparticles used for the in vivo imaging of subcutaneous tumors. Biochim Biophys Acta. 2017;1861(6):1587-96. https://doi.org/10.1016/j.bbagen.2017.01.036.

Alivisatos A. Semiconductor clusters, nanocrystals, and quantum dots. Science. 1996;271(5251):933-7. https://doi. org/10.1126/science.271.5251.933.

Allen T, Cullis P. Liposomal drug delivery systems: from concept to clinical applications. Adv Drug Deliv Rev. 2013;65:3648. https://doi.org/10.1016/j.addr.2012.09.037.

Alric C, Aubrey N, Allard-Vannier É, et al. Covalent conjugation of cysteine-engineered scFv to PEGylated magnetic nanoprobes for immunotargeting of breast cancer cells. RSC Adv. 2016;6:37099-109. https://doi.org/10.1039/ c6ra06076e.

An Y, Chen M, Xue Q, et al. Preparation and self-assembly of carboxylic acid-functionalized silica. J Colloid Interface Sci. 2007;311(2):507-13. https://doi.org/10.1016/j.jcis.2007.02.084.

Anselmo A, Mitragotri S. A review of clinical translation of inorganic nanoparticles. AAPS J. 2015;17:1041-54. https://doi. org/10.1208/s12248-015-9780-2.

Arriagada F, Osseo-Asare K. Synthesis of nanosize silica in aerosol OT reverse microemulsions. J Colloid Interface Sci. 1995;170(1):8-17. https://doi.org/10.1006/jcis.1995.1064.

Arriagada F, Osseo-Asare K. Controlled hydrolysis of tetraethoxysilane in a nonionic water-in-oil microemulsion: a statistical model of silica nucleation. J Colloids Surf A. 1999a;154:311-26. https://doi.org/10.1016/s0927-7757(98)00870-x.

Arriagada F, Osseo-Asare K. Synthesis of nanosize silica in a nonionic water-in-oil microemulsion effects of the water/ surfactant molar ratio and ammonia concentration. J Colloids Interface Sci. 1999b;211:210-20. https://doi. org/10.1006/jcis.1998.5985.

Ash C, Dubec M, Donne K, et al. Effect of wavelength and beam width on penetration in light-tissue interaction using computational methods. Lasers Med Sci. 2017;32(8):1909-18. https://doi.org/10.1007/s10103-017-2317-4.

Bae S, Tan W, Hong J. Fluorescent dye-doped silica nanoparticles: new tools for bioapplications. Chem Commun. 2012;48:2270-82. https://doi.org/10.1039/c2cc16306c.

Bagwe R, Yang C, Hilliard L, et al. Optimisation of dye-doped silica nanoparticles prepared using a reverse microemulsion method. Langmuir. 2004;20:8336-42. https://doi.org/10.1021/la049137j.

Bagwe R, Hilliard L, Tan W. Surface modification of silica nanoparticles to reduce aggregation and non-specific binding. Langmuir. 2006;22(9):4357-62. https://doi.org/10.1021/la052797j.

Banerjee R, Jaiswal A. Recent advances in nanoparticle-based lateral flow immunoassay as a point-of-care diagnostic tool for infectious agents and diseases. Analyst. 2018;143:1970-96. https://doi.org/10.1039/c8an00307f.

Barandeh F, Nguyen P, Kumar R, et al. Organically modified silica nanoparticles are biocompatible and can be targeted to neurons in vivo. PLoS ONE. 2012;7(1):e29424. https://doi.org/10.1371/journal.pone.0029424.

Barbu E, Molnàr É, Tsibouklis J, et al. The potential for nanoparticle-based drug delivery to the brain: overcoming the blood-brain barrier. Expert Opin Drug Deliv. 2009;6(6):553-65. https://doi.org/10.1517/17425240902939143.

Bartolome J, Echegoyen L, Fragoso A. Reactive Carbon nano-onion modified glassy carbon surfaces as DNA Sensors for human papillomavirus oncogene detection with enhanced sensitivity. Anal Chem. 2015;87(13):6744-51. https:// doi.org/10.1021/acs.analchem.5b00924.

Battistelli G, Cantelli A, Guidetti G, et al. Ultra-bright and stimuli-responsive fluorescent nanoparticles for bioimaging. WIREs Nanomed Nanobiotechnol. 2016;8:139-50. https://doi.org/10.1002/wnan.1351.

Beck G, Ha S, Camalier C, et al. bioactive silica-based nanomaterials stimulate bone-forming osteoblasts, suppress boneresorbing osteoclasts, and enhance bone mineral density in vivo. Nanomed Nanotechnol Biol Med. 2012;8(6):793803. https://doi.org/10.1016/j.nano.2011.11.003.

Benachour H, Sève A, Bastogne T, et al. Multifunctional peptide-conjugated hybrid silica nanoparticles for photodynamic therapy and MRI. Theranostics. 2012;2(9):889-904. https://doi.org/10.7150/thno.4754.

Benezra M, Penate-Medina O, Zanzonico P, et al. Multimodal silica nanoparticles are effective cancer-targeted probes in a model of human melanoma. J Clin Invest. 2011;121(7):2768-80. https://doi.org/10.1172/jci45600.

Biffi S, Petrizza L, Rampazzo E, et al. Multiple dye-doped NIR-emitting silica nanoparticles for both flow cytometry and in vivo imaging. RSC Adv. 2014;4:18278. https://doi.org/10.1039/c4ra01535e.

Biju V. Chemical modifications and bioconjugate reactions of nanomaterials for sensing, imaging, drug delivery and therapy. Chem Soc Rev. 2014;43:744-64. https://doi.org/10.1039/c3cs60273g.

Bogush G, Zukoski C. Uniform silica particle precipitation: an aggregative growth model. J Colloid Interface Sci. 1992;142:19-34. https://doi.org/10.1016/0021-9797(91)90030-c.

Bohne-Lang A, von der Lieth C. GlyProt: in silico glycosylation of proteins. Nucleic Acids Res. 2005;33:W214-9. https://doi. org/10.1093/nar/gki385.

Bonacchi S, Genovese D, Juris R, et al. Luminescent silica nanoparticles: extending the frontiers of brightness. Angew Chem Int Ed. 2011;50:4056-66. https://doi.org/10.1002/anie.201004996.

Boogerd L, Boonstra M, Beck A, et al. Concordance of folate receptor-a expression between biopsy, primary tumor and metastasis in breast cancer and lung cancer patients. Oncotarget. 2016;7(14):17442-54. https://doi.org/10.18632/ oncotarget.7856.

Bouwmeester $\mathrm{H}$, van der Zande $\mathrm{M}$, Jepson M. Effects of food-borne nanomaterials on gastrointestinal tissues and microbiota. Wiley Interdiscip Rev Nanomed Nanobiotechnol. 2018;10(1):e1481. https://doi.org/10.1002/wnan.1481.

Burns A, Ow H, Wiesner U. Fluorescent core-shell silica nanoparticles: towards "Lab on a Particle" architectures for nanotechnology. Chem Soc Rev. 2006;35(11):1028-42. https://doi.org/10.1039/b600562b.

Butkevich A, Lukinavičius G, D’Este E, et al. Cell-permeant large stokes shift dyes for transfection-free multicolor nanoscopy. J Am Chem Soc. 2017;139(36):12378-81. https://doi.org/10.1021/jacs.7b06412.

Cai C, Lin J. Self-assembly: served on a nanoplate. Nat Chem. 2014;6:857-8. https://doi.org/10.1038/nchem.2071.

Cao F, Deng R, Liu D, et al. Fabrication of fluorescent silica-Au hybrid nanostructures for targeted imaging of tumor cells. Dalton Trans. 2011;40:4800. https://doi.org/10.1039/c1dt10106d.

Carrillo-Carrion C, Carril M, Parak W. Techniques for the experimental investigation of the protein corona. Curr Opin Biotechnol. 2017;46:106-13. https://doi.org/10.1016/j.copbio.2017.02.009. 
Chang H, Yeh M. Clinical development of liposome-based drugs: formulation, characterisation, and therapeutic efficacy. Int J Nanomed. 2012;7:49-60. https://doi.org/10.2147/ijn.s26766.

Chatziharalambous D, Lygirou V, Latosinska A, et al. Analytical performance of ELISA assays in urine: one more bottleneck towards biomarker validation and clinical implementation. PLoS ONE. 2016;11(2):e0149471. https://doi. org/10.1371/journal.pone.0149471.

Chen Y, Zhang Y. Fluorescent quantification of amino groups on silica nanoparticle surfaces. Anal Bioanal Chem. 2011;399(7):2503-9. https://doi.org/10.1007/s00216-010-4622-7.

Chen J, Wang D, Xi J, et al. Immune gold nanocages with tailored optical properties for targeted photothermal destruction of cancer cells. Nano Lett. 2007;7(5):1318-22. https://doi.org/10.1021/nl070345g.

Chen H, Ahsan S, Santiago-Berrios M, et al. Mechanisms of quenching of alexa fluorophores by natural amino acids. J Am Chem Soc. 2010;132(21):7244-5. https://doi.org/10.1021/ja100500k.

Cheng Z, Al Zaki A, Hui J, et al. Multifunctional nanoparticles: cost versus benefit of adding targeting and imaging capabilities. Science. 2012;338:903. https://doi.org/10.1126/science.1226338.

Chetwynd A, Wheeler K, Lynch I. Best practice in reporting corona studies: minimum information about Nanomaterial Biocorona Experiments (MINBE). Nano Today. 2019. https://doi.org/10.1016/..nantod.2019.06.004.

Chunduri L, Kurdekar A, Haleyurgirisetty M, et al. Femtogram level sensitivity achieved by surface engineered silica nanoparticles in the early detection of HIV infection. Sci Rep. 2017;7:7149. https://doi.org/10.1038/s41598-017-07299-1.

Claesson E, Philipse A. Thiol-functionalized silica colloids, grains, and membranes for irreversible adsorption of metal(oxide) nanoparticles. Colloids Surf A Physicochem Eng Aspects. 2007;297:46-54. https://doi.org/10.1016/j. colsurfa.2006.10.019.

Cockburn A, Bradford R, Buck N, et al. Approaches to the safety assessment of engineered nanomaterials (ENM) in food. Food Chem Toxicol. 2012;50(6):2224-42. https://doi.org/10.1016/.fft.2011.12.029.

Corbo C, Molinaro R, Parodi A, et al. The impact of nanoparticle protein corona on cytotoxicity, immunotoxicity and target drug delivery. Nanomed Nanotechnol Biol Med. 2016;11(1):81-100. https://doi.org/10.2217/nnm.15.188.

Danhier F, Feron O, Préat V. To exploit the tumor microenvironment: passive and active tumor targeting of nanocarriers for anti-cancer drug delivery. J Control Release. 2010;148:135-46. https://doi.org/10.1016/j.jconrel.2010.08.027.

de Lara L, Michelon M, Metin C, et al. Interface tension of silica hydroxylated nanoparticle with brine: a combined experimental and molecular dynamics study. J Chem Phys. 2012;136:164702.

de Lara L, Rigo V, Michelon M, et al. Molecular dynamics studies of aqueous silica nanoparticle dispersions: salt effects on the double layer formation. J Phys Condens Matter. 2015;27(32):325101. https://doi.org/10.1088/09538984/27/32/325101.

Desmettre T, Devoisselle J, Mordon S. Fluorescence properties and metabolic features of indocyanine green (ICG) as related to angiography. Surv Ophthalmol. 2000;45:15-27. https://doi.org/10.1016/s0039-6257(00)00123-5.

Detappe A, Kunjachan S, Sancey L, et al. Advanced multimodal nanoparticles delay tumor progression with clinical radiation therapy. J Control Release. 2016;238:103-13. https://doi.org/10.1016/j.jconrel.2016.07.021.

Dhir A, Datta A. FRET on surface of silica nanoparticle: effect of chromophore concentration on dynamics and efficiency. J Phys Chem C. 2016;120(36):20125-31. https://doi.org/10.1021/acs.jpcc.6b05242.

Dixon S, Lemberg K, Lamprecht M, et al. Ferroptosis: an iron-dependent form of nonapoptotic cell death. Cell. 2012;149(5):1060-72. https://doi.org/10.1016/j.cell.2012.03.042.

Ducray A, Felser A, Zielinski J, et al. Effects of silica nanoparticle exposure on mitochondrial function during neuronal differentiation. J Nanobiotechnol. 2017;15:49. https://doi.org/10.1186/s12951-017-0284-3.

Dufort S, Bianchi A, Henry M, et al. Nebulized gadolinium-based nanoparticles: a theranostic approach for lung tumor imaging and radiosensitization. Small. 2014;11(2):215-21.

European Commission (2015) Guidance on the determination of potential health effects of nanomaterials used in medical devices. Scientific Committee on Emerging and Newly Identified Health Risks-Opinion. https://doi. org/10.2772/41391.

Fang J, Nakamura $\mathrm{H}$, Maeda $\mathrm{H}$. The EPR effect: unique features of tumor blood vessels for drug delivery, factors involved, and limitations and augmentation of the effect. Adv Drug Deliv Rev. 2011;63:136-51. https://doi. org/10.1016/j.addr.2010.04.009

Farka Z, Juř́k T, Kováŕ D, et al. Nanoparticle-based immunochemical biosensors and assays: recent advances and challenges. Chem Rev. 2017;117(15):9973-10042. https://doi.org/10.1021/acs.chemrev.7b00037.

Finnie K, Bartlett J, Barbe C, et al. Formation of silica nanoparticles in microemulsions. Langmuir. 2007;23(6):3017-24. https://doi.org/10.1021/la0624283.

Francia V, Yang K, Deville S, et al. Corona composition can affect the mechanisms cells use to internalize nanoparticles. ACS Nano. 2019. https://doi.org/10.1021/acsnano.9b03824

Fruijtier-Pölloth C. The safety of nanostructured synthetic amorphous silica (SAS) as a food additive (E 551). Arch Toxicol. 2016;90(12):2885-916. https://doi.org/10.1007/s00204-016-1850-4.

Galluzzi L, et al. Molecular mechanisms of cell death: recommendations of the Nomenclature Committee on Cell Death. Cell Death Differ. 2018;25(3):486-541. https://doi.org/10.1038/s41418-017-0012-4.

Gao X, Yang L, Petros J, et al. In vivo molecular and cellular imaging with quantum dots. Curr Opin Biotechnol. 2005;16(1):63-72. https://doi.org/10.1016/j.copbio.2004.11.003.

Giovannini G, De Angelis F. Novel electro-magnetophoretic separation method for the highly sensitive detection of analytes. Nanoscale Horizons. 2019. https://doi.org/10.1039/c9nh00279k.

Giovannini G, Kunc F, Piras C, et al. Stabilizing silica nanoparticles in hydrogels: impact on storage and polydispersity. RSC Adv. 2017;7:19924-33. https://doi.org/10.1039/c7ra02427d.

Giovannini G, Moore C, Hall A, et al. pH-Dependent silica nanoparticle dissolution and cargo release. Coll Surf B Biointerfaces. 2018a;169:242-8. https://doi.org/10.1016/j.colsurfb.2018.04.064.

Giovannini G, Warncke P, Fischer D, et al. Improving colloidal stability of silica nanoparticles when stored in responsive gel: application and toxicity study. Nanotoxicology. 2018b;12(5):407-22. https://doi.org/10.1080/17435 390.2018.1457729. 
Gnanasammandhan M, Idris N, Bansal A, et al. Near-IR photoactivation using mesoporous silica-coated NaYF4:Yb, Er/ Tm upconversion nanoparticles. Nat Prot. 2016;11(4):688-713. https://doi.org/10.1038/nprot.2016.035.

Gorman A, Killoran J, O'Shea C, et al. In vitro demonstration of the heavy-atom effect for photodynamic therapy. J Am Chem Soc. 2004;126:10619-31. https://doi.org/10.1021/ja047649e.

Gubala V, Le Gueval X, Nooney R, et al. A comparison of mono and multivalent linkers and their effect on the colloidal stability of nanoparticle and immunoassays performance. Talanta. 2010;81(4-5):1833-9. https://doi. org/10.1016/j.talanta.2010.03.048.

Gubala V, Lynam C, Nooney R, et al. Kinetics of immunoassays with particles as labels: effect of antibody coupling using dendrimers as linkers. Analyst. 2011;136(12):2533-41. https://doi.org/10.1039/c1an15017k.

Gubala V, Harris L, Ricco A, et al. Point of care diagnostics: status and future. Anal Chem. 2012;84:487-515. https://doi. org/10.1021/ac203019.

Gubala V, Johnston L, Krug H, et al. Engineered nanomaterials and human health: Part 2. applications and nanotoxicology (IUPAC Technical Report). Pure Appl Chem. 2018a;90(8):1325-56. https://doi.org/10.1515/ pac-2017-0102.

Gubala V, Johnson L, Liu Z, et al. Engineered nanomaterials and human health: part 1. Preparation, functionalization and characterization (IUPAC Technical Report). Pure Appl Chem. 2018b;90(8):1283-324. https://doi. org/10.1515/pac-2017-0101.

Ha S, Weitzmann M, Beck G. Bioactive silica nanoparticles promote osteoblast differentiation through stimulation of autophagy and direct association with LC3 and p62. ACS Nano. 2014;8(6):5898-910. https://doi.org/10.1021/ nn5009879.

Hagemans F, van der Wee E, van Blaaderen A, et al. Synthesis of cone-shaped colloids from rod-like silica colloids with a gradient in the etching rate. Langmuir. 2016;32(16):3970-6. https://doi.org/10.1021/acs.langmuir.6b006 78.

Hagemans F, Vlug W, Raffaelli C, et al. Sculpting silica colloids by etching particles with nonuniform compositions. Chem Mater. 2017;29(7):3304-13. https://doi.org/10.1021/acs.chemmater.7b00687.

Hainfeld J, Slatkin D, Smilowitz H. The use of gold nanoparticles to enhance radiotherapy in mice. Phys Med Biol. 2004:49(18):N309-15. https://doi.org/10.1088/0031-9155/49/18/n03.

Halamoda-Kenzaoui B, Ceridono M, Urbán P, et al. The agglomeration state of nanoparticles can influence the mechanism of their cellular internalisation. J Nanobiotechnol. 2017;15:48. https://doi.org/10.1186/s12951-017-0281-6.

Halamoda-Kenzaoui B, Holzwarth U, Roebben G, et al. Mapping of the available standards against the regulatory needs for nanomedicines. Wiley Interdiscip Rev Nanomed Nanobiotechnol. 2019;11(1):e1531. https://doi.org/10.1002/ wnan.1531.

Hameed S, Chen $\mathrm{H}$, Irfan M, et al. Fluorescence guided sentinel lymph node mapping: from current molecular probes to future multimodal nanoprobes. Bioconjug Chem. 2019;30(1):13-28. https://doi.org/10.1021/acs.bioconjche m.8b00812.

Hardy A, Benford D, Halldorsson T, et al. Guidance on risk assessment of the application of nanoscience and nanotechnologies in the food and feed chain: part 1, human and animal health. EFSA J. 2018;16(7):5327. https://doi. org/10.2903/j.efsa.2018.5327.

Hartlen K, Athanasopoulos A, Kitaev V. Facile preparation of highly monodisperse small silica spheres (15 to > $200 \mathrm{~nm}$ ) suitable for colloidal templating and formation of ordered arrays. Langmuir. 2008;24(5):1714-20. https://doi. org/10.1021/la7025285.

Hayashi K, Nakamura M, Miki H, et al. Photostable iodinated silica/porphyrin hybrid nanoparticles with heavy-atom effect for wide-field photodynamic/photothermal therapy using single light source. Adv Funct Mater. 2014;24(4):503-13. https://doi.org/10.1002/adfm.201301771

He X, Nie H, Wang K, et al. In vivo study of biodistribution and urinary excretion of surface-modified silica nanoparticles. Anal Chem. 2008;80(24):9597-603. https://doi.org/10.1021/ac801882g.

Henderson T, Morries L. Near-infrared photonic energy penetration: can infrared phototherapy effectively reach the human brain? Neuropsychiatr Dis Treat. 2015;11:2191-208. https://doi.org/10.2147/ndt.578182.

Herda L, Hristov D, Lo Giudice M, et al. Mapping of molecular structure of the nanoscale surface in bionanoparticles. J Am Chem Soc. 2017;139(1):111-4. https://doi.org/10.1021/jacs.6b12297.

Hermanson G. Bioconjugate techniques. 3rd ed. Amsterdam: Elsevier; 2013. https://doi.org/10.1016/c2009-0-64240-9.

Herz E, Burns A, Bonner D, et al. Large stokes-shift fluorescent silica nanoparticles with enhanced emission over free dye for single excitation multiplexing. Macromol Rapid Commun. 2009;30(22):1907-10. https://doi.org/10.1002/ marc.200900389.

Hildebrandt N, Tagit O. Colloidal nanoparticles for signal enhancement in optical diagnostic assays. J Nanosci Nanotechnol. 2018;18(10):6671-9. https://doi.org/10.1166/jnn.2018.15748.

Hong B, Panagiotopoulos A. Molecular dynamics simulations of silica nanoparticles grafted with poly(ethylene oxide) oligomer chains. J Phys Chem B. 2012;116:2385-95. https://doi.org/10.1021/jp2112582.

Hong N, Kim H, Lee H, et al. Fluorescent property of indocyanine green (ICG) rubber ring using LED and laser light sources. Biomed Opt Express. 2016;7(5):1637-44. https://doi.org/10.1364/boe.7.001637.

Hristov D, Mahon E, Dawson K. Controlling aqueous silica nanoparticle synthesis in the 10-100 nm range. Chem Commun. 2015a;51:17420-3. https://doi.org/10.1039/c5cc06598d.

Hristov D, Rocks L, Kelly P, et al. Tuning of nanoparticle biological functionality through controlled surface chemistry and characterisation at the bioconjugated nanoparticle surface. Sci Rep. 2015b;5:17040. https://doi.org/10.1038/srep1 7040 .

Hsiao I, Fritsch-Decker S, Leidner A, et al. Biocompatibility of amine-functionalized silica nanoparticles: the role of surface coverage. Small. 2019. https://doi.org/10.1002/smll.201805400.

Hu H, Li Q, Jiang L, et al. Genome-wide transcriptional analysis of silica nanoparticle-induced toxicity in zebrafish embryos. Toxicol Res. 2016;5:609-20. https://doi.org/10.1039/c5tx00383k.

Huang Y, Pemberton J. Synthesis of uniform, spherical sub-100 nm silica particles using a conceptual modification of the classic LaMer model. Coll Surf A. 2010;360:175-80. https://doi.org/10.1016/j.colsurfa.2010.02.031. 
Huo Q, Liu J, Wang L, et al. A new class of silica cross-linked micellar core-shell nanoparticles. J Am Chem Soc. 2006;128(19):6447-53. https://doi.org/10.1021/ja060367p.

Imhof A, Megens M, Engelberts J, et al. Spectroscopy of fluorescein (FITC) dyed colloidal silica spheres. J Phys Chem B. 1999;103:1408. https://doi.org/10.1021/jp983241q.

Ishida T, Kiwada H. Accelerated blood clearance (ABC) phenomenon upon repeated injection of PEGylated liposomes. Int J Pharm. 2008;354:56-62. https://doi.org/10.1016/j.jpharm.2007.11.005.

Jain S, Coulter J, Hounsell A, et al. Cell-specific radiosensitization by gold nanoparticles at megavoltage radiation energies. Int J Radiat Oncol Biol Phys. 2011;79(2):531-9. https://doi.org/10.1016/j.jijobp.2010.08.044.

Jayawardana K, Wijesundera S, Yan M. Aggregation-based detection of M. smegmatis using D-arabinose-functionalized fluorescent silica nanoparticles. Chem Commun. 2015;51:15964-6. https://doi.org/10.1039/c5cc05772h.

Jeong S, Park J, Cha M, et al. Highly robust and optimized conjugation of antibodies to nanoparticles using quantitatively validated protocols. Nanoscale. 2017;9(7):2548-55. https://doi.org/10.1039/c6nr04683e.

Jung H, Moon D, Lee J. Quantitative analysis and efficient surface modification of silica nanoparticles. J Nanomater. 2012. https://doi.org/10.1155/2012/593471.

Kadimisetty K, Malla S, Sardesai N, et al. Automated multiplexed ECL immunoarrays for cancer biomarker proteins. Anal Chem. 2015;87(8):4472-8. https://doi.org/10.1021/acs.analchem.5b00421.

Katt M, Placone A, Wong A, et al. In Vitro tumor models: advantages, disadvantages, variables, and selecting the right platform. Front Bioeng Biotechnol. 2016:4:12. https://doi.org/10.3389/fbioe.2016.00012.

Ke R, Yang W, Xia X, et al. Tandem conjugation of enzyme and antibody on silica nanoparticle for enzyme immunoassay. Anal Biochem. 2010;406(1):8-13. https://doi.org/10.1016/j.ab.2010.06.039.

Ke P, Lin S, Parak W, et al. A decade of the protein corona. ACS Nano. 2017;11(12):11773-6. https://doi.org/10.1021/acsna no.7b08008.

Kelleher S, Nooney R, Flynn S, et al. Multivalent linkers for improved covalent binding of oligonucleotides to dye-doped silica nanoparticles. Nanotechnology. 2015;26:365703. https://doi.org/10.1088/0957-4484/26/36/365703.

Kelly P, Åberg C, Polo E, et al. Mapping protein binding sites on the biomolecular corona of nanoparticles. Nat Nanotechnol. 2015;10:472-9. https://doi.org/10.1038/nnano.2015.47.

Kempen P, Greasley S, Parker K, et al. Theranostic mesoporous silica nanoparticles biodegrade after pro-survival drug delivery and ultrasound/magnetic resonance imaging of stem cells. Theranostics. 2015;5(6):631-42. https://doi. org/10.7150/thno.11389.

Kim S, Ohulchanskyy T, Pudavar H, et al. Organically modified silica nanoparticles co-encapsulating photosensitizing drug and aggregation-enhanced two-photon absorbing fluorescent dye aggregates for two-photon photodynamic therapy. J Am Chem Soc. 2007;129(9):2669-75. https://doi.org/10.1021/ja0680257.

Kim I, Joachim E, Choi H, et al. Toxicity of silica nanoparticles depends on size, dose, and cell type. Nanomed Nanotechnol Biol Med. 2015;11(6):1407-16. https://doi.org/10.1016/j.nano.2015.03.004.

Kitai T, Inomoto T, Miwa M, et al. Fluorescence navigation with indocyanine green for detecting sentinel lymph nodes in breast cancer. Breast Cancer. 2005;12:211-5. https://doi.org/10.2325/jbcs.12.211.

Kobayashi Y, Horie M, Konno M, et al. Preparation and properties of silica-coated cobalt nanoparticles. J Phys Chem B. 2003;107(30):7420-5. https://doi.org/10.1021/jp027759c.

Koch F, Moller A, Frenz M, et al. An in vitro toxicity evaluation of gold-, PLLA- and PCL-coated silica nanoparticles in neuronal cells for nanoparticle-assisted laser-tissue soldering. Toxicol In Vitro. 2014;28(5):990-8. https://doi. org/10.1016/j.tiv.2014.04.010.

Koo Y, Cao Y, Kopelman R, et al. Real-time measurements of dissolved oxygen inside live cells by organically modified silicate fluorescent nanosensors. Anal Chem. 2004;76(9):2498-505. https://doi.org/10.1021/ac035493f.

Korzeniowska B, Nooney R, Wencel D, et al. Silica nanoparticles for cell imaging and intracellular sensing. Nanotechnology. 2013;24:442002. https://doi.org/10.1088/0957-4484/24/44/442002.

Korzeniowska B, Woolley R, DeCourcey J, et al. Intracellular pH-sensing using core/shell silica nanoparticles. J Biomed Nanotechnol. 2014;10(7):1336-45. https://doi.org/10.1166/jbn.2014.1815.

Krug H. Nanosafety research—are we on the right track? Angew Chem Int Ed. 2014;53:12304-19. https://doi. org/10.1002/anie.201403367.

Ku S, Yan F, Wang Y, et al. The blood-brain barrier penetration and distribution of PEGylated fluorescein-doped magnetic silica nanoparticles in rat brain. Biochem Biophys Res Commun. 2010;394:871-6. https://doi.org/10.1016/j. bbrc.2010.03.006.

Kuijk A, van Blaaderen A, Imhof A. Synthesis of monodisperse, rodlike silica colloids with tunable aspect ratio. J Am Chem Soc. 2011;133(8):2346-9. https://doi.org/10.1021/ja109524h.

Kumar R, Roy I, Ohulchanskky T, et al. In vivo biodistribution and clearance studies using multimodal organically modified silica nanoparticles. ACS Nano. 2010;4(2):699-708. https://doi.org/10.1021/nn901146y.

Kunc F, Balhara V, Brinkmann A, et al. Quantification and stability determination of surface amine groups on silica nanoparticles using solution NMR. Anal Chem. 2018;90(22):13322-30. https://doi.org/10.1021/acs.analchem.8b02803.

Kunc F, Moore C, Sully R, et al. Poly-carboxylated dextran as a multivalent crosslinker: synthesis and target recognition of the antibody-nanoparticle bioconjugates in PBS and serum. Langmuir. 2019. https://doi.org/10.1021/acs.langm uir.8b03833.

LaMer V, Dinegar R. Theory, production and mechanism of formation of monodispersed hydrosols. J Am Chem Soc. 1950;72:4847-54. https://doi.org/10.1021/ja01167a001.

Lara S, Alnasser F, Polo E, et al. Identification of receptor binding to the biomolecular corona of nanoparticles. ACS Nano. 2017;11:1884-93. https://doi.org/10.1021/acsnano.6b07933.

Lee J, Jun Y, Yeon S, et al. Dual-mode nanoparticle probes for high-performance magnetic resonance and fluorescence imaging of neuroblastoma. Angew Chem Int Ed. 2006;455:8160. https://doi.org/10.1002/anie.200603052.

Lee J, Lee N, Kim H, et al. Uniform mesoporous dye-doped silica nanoparticles decorated with multiple magnetite nanocrystals for simultaneous enhanced magnetic resonance imaging, fluorescence imaging, and drug delivery. $J$ Am Chem Soc. 2010;132(2):552-7. https://doi.org/10.1021/ja905793q. 
Lee Y, Choi E, Webster T, et al. Effect of the protein corona on nanoparticles for modulating cytotoxicity and immunotoxicity. Int J Nanomed. 2015;10:97-113. https://doi.org/10.2147/ijn.s72998.

Li Y, Xu W. Highly sensitive detection of Shigella flexneri using fluorescent silica nanoparticles. New Microbial. 2009;32(4):377-83.

Li N, Zeng S, He L, et al. Probing nanoparticle-protein interaction by capillary electrophoresis. Anal Chem. 2010;82(17):7460-6. https://doi.org/10.1021/ac101627p.

Li Z, Barnes J, Bosoy A, et al. Mesoporous silica nanoparticles in biomedical applications. Chem Soc Rev. 2012;41:2590605. https://doi.org/10.1039/c1cs15246g

Li H, Mu Y, Qian S, et al. Synthesis of fluorescent dye-doped silica nanoparticles for target-cell-specific delivery and intracellular MicroRNA imaging. Analyst. 2014;40(2):567-73. https://doi.org/10.1039/c4an01706d.

Liberman A, Mendez N, Trogler W, et al. Synthesis and surface functionalization of silica nanoparticles for nanomedicine. Surf Sci Rep. 2014;69(2):132-58. https://doi.org/10.1016/j.surfrep.2014.07.001.

Liu J, Bai S, Zhong H, et al. Tunable assembly of organosilica hollow nanospheres. J Phys Chem C. 2009;114:953-61. https ://doi.org/10.1021/jp909931z.

Liu F, Fang F, Yuan H, et al. Suppression of autophagy by FIP200 deletion leads to osteopenia in mice through the inhibition of osteoblast terminal differentiation. J Bone Miner Res. 2013;28(11):2414-30. https://doi.org/10.1002/ jbmr.1971.

Liu Y, Wang X, Song W, et al. Synthesis and characterization of silica nanoparticles functionalized with multiple TEMPO groups and investigation on their oxidation activity. Polym Chem. 2015;6:7514-23. https://doi.org/10.1039/c5py0 $1190 f$.

Longbottom B, Rochford L, Beanland R, et al. Mechanistic insight into the synthesis of silica-based "Matchstick" colloids. Langmuir. 2015;31(33):9017-25. https://doi.org/10.1021/acs.langmuir.5b02645.

Lu H. Synthesis and characterization of amino-functionalized silica nanoparticles. Colloid J. 2013;75(3):311-8. https://doi. org/10.1134/s1061933×13030125.

Lu X, Qian J, Zhou H, et al. In vitro cytotoxicity and induction of apoptosis by silica nanoparticles in human HepG2 hepatoma cells. Int J Nanomed. 2011;6:1889-901. https://doi.org/10.2147/ijn.s24005.

Lukianova-Hleb E, Belyanin A, Kashinath S, et al. Plasmonic nanobubble-enhanced endosomal escape processes for selective and guided intracellular delivery of chemotherapy to drug-resistant cancer cells. Biomaterials. 2012;33(6):1821-6. https://doi.org/10.1016/j.biomaterials.2011.11.015.

Lux F, Tran V, Thomas E, et al. AGulX ${ }^{\circledR}$ from bench to bedside-transfer of an ultrasmall theranostic gadolinium-based nanoparticle to clinical medicine. Br J Radiol. 2019;92:2018036. https://doi.org/10.1259/bjr.20180365.

Ma Y, Li Y, Ma S, et al. Highly bright water-soluble silica coated quantum dots with excellent stability. J Mater Chem B. 2014;2:5043-51. https://doi.org/10.1039/c4tb00458b.

Ma K, Mendoza C, Hanson M, et al. Control of ultrasmall Sub10nm ligand-functionalised fluorescent core-shell silica nanoparticle growth in water. Chem Mater. 2015;27:4119-33. https://doi.org/10.1021/acs.chemmater.5b01222.

Ma K, Zhang D, Cong Y, et al. Elucidating the mechanism of silica nanoparticle PEGylation processes using fluorescence correlation spectroscopies. Chem Mater. 2016;28(5):1537-45. https://doi.org/10.1021/acs.chemmater.6b00030.

Mahon E, Salvati A, Bombelli F, et al. Designing the nanoparticle-biomolecule interface for targeting and therapeutic delivery. J Control Release. 2012a;161(2):164-74. https://doi.org/10.1016/j.jconrel.2012.04.009.

Mahon E, Hristov D, Dawson K. Stabilising fluorescent silica nanoparticles against dissolution effects for biological studies. Chem Commun. 2012b;48:7970-2. https://doi.org/10.1039/c2cc34023b.

Maiolo D, Bergese P, Mahon E, et al. Surfactant titration of nanoparticle-protein corona. Anal Chem. 2014;86(24):1205563. https://doi.org/10.1021/ac5027176.

Malba C, Sudhakaran U, Borsacchi S, et al. Structural and photophysical properties of rare-earth complexes encapsulated into surface modified mesoporous silica nanoparticles. Dalton Trans. 2014;43:16183-96. https://doi.org/10.1039/ c4dt00760c.

Maltez-da Costa M, de la Escosura-Muñiz A, Nogués C, et al. Detection of circulating cancer cells using electrocatalytic gold nanoparticles. Small. 2012;8(23):3605-12. https://doi.org/10.1002/smll.201201205.

Matsoukas T, Gulari E. Monomer-addition growth with a slow initiation step: a growth model for silica particles from alkoxides. J Colloid Interface Sci. 1989;132:13-21. https://doi.org/10.1016/0021-9797(89)90210-5.

McCusker L, Liebau F, Engelhardt G. Nomenclature of structural and compositional characteristics of ordered microporous and mesoporous materials with inorganic hosts (IUPAC Recommendations 2001). Pure Appl Chem. 2001;73(2):381-94. https://doi.org/10.1351/pac200173020381.

McMahon S, Hyland W, Muir M, et al. Biological consequences of nanoscale energy deposition near irradiated heavy atom nanoparticles. Sci Rep. 2011:1:18. https://doi.org/10.1038/srep00018.

Mech A, Rasmussen K, Jantunen P, et al. Insights into possibilities for grouping and read-across for nanomaterials in EU chemicals legislation. Nanotoxicology. 2019;13(1):119-41. https://doi.org/10.1080/17435390.2018.1513092.

Meder F, Thomas S, Fitzpatrick L, et al. Labeling the structural integrity of nanoparticles for advanced in situ tracking in bionanotechnology. ACS Nano. 2016;10(4):4660-71. https://doi.org/10.1021/acsnano.6b01001.

Miernicki M, Hofmann T, Eisenberger I, et al. Legal and practical challenges in classifying nanomaterials according to regulatory definitions. Nat Nanotechnol. 2019;14:208-16. https://doi.org/10.1038/s41565-019-0396-z.

Miletto I, Bottinelli E, Caputo G, et al. Bright photoluminescent hybrid mesostructured silica nanoparticles. Phys Chem Chem Phys. 2012;14(28):10015-21. https://doi.org/10.1039/c2cp40975e.

Miot-Noirault E, Vidal A, Morlieras J, et al. Small rigid platforms functionalization with quaternary ammonium: targeting extracellular matrix of chondrosarcoma. Nanomed Nanotechnol Biol Med. 2014;10(8):1887-95. https://doi. org/10.1016/j.nano.2014.06.011.

Mohajeri M, Brummer R, Rastall R, et al. The role of the microbiome for human health: from basic science to clinical applications. Eur J Nutr. 2018;57:1-14. https://doi.org/10.1007/s00394-018-1703-4.

Monopoli M, Walczyk D, Campbell A, et al. Physical-chemical aspects of protein corona: relevance to in vitro and in vivo biological impacts of nanoparticles. J Am Chem Soc. 2011;133(8):2525-34. https://doi.org/10.1021/ja107583h. 
Monopoli M, Åberg C, Salvati A, et al. Biomolecular coronas provide the biological identity of nanosized materials. Nat Nanotechnol. 2012;7:779-86. https://doi.org/10.1038/nnano.2012.207.

Montalti M, Prodi L, Rampazzo E, et al. Dye-doped silica nanoparticles as luminescent organized systems for nanomedicine. Chem Soc Rev. 2014;43:4243. https://doi.org/10.1039/c3cs60433k.

Montón H, Nogués C, Rossinyol E, et al. QDs versus Alexa: reality of promising tools for immunocytochemistry. J Nanobiotechnol. 2009;7:4. https://doi.org/10.1186/1477-3155-7-4.

Montón H, Roldán M, Merkoçi A, et al. The use of quantum dots for immunochemistry applications. Nanopart Biol Med. 2012. https://doi.org/10.1007/978-1-61779-953-2_13.

Moore C, Montón H, O'Kennedy R, et al. Controlling colloidal stability of silica nanoparticles during bioconjugation reactions with proteins and improving their longer-term stability, handling and storage. J Mater Chem B. 2015;3:204355. https://doi.org/10.1039/c4tb01915f.

Moore C, Giovannini G, Kunc F, et al. 'Overloading'fluorescent silica nanoparticles with dyes to improve biosensor performance. J Mater Chem B. 2017;5:5564-72. https://doi.org/10.1039/c7tb01284e.

Mordon S, Devoisselle J, Soulie-Begu S, et al. Indocyanine green: physicochemical factors affecting its fluorescence in vivo. Microvasc Res. 1998;55:146-52. https://doi.org/10.1006/mvre.1998.2068.

Moremen K, Tiemeyer M, Nairn A. Vertebrate protein glycosylation: diversity, synthesis and function. Rev Mol Cell Biol. 2012;13(7):448-62. https://doi.org/10.1038/nrm3383.

Murugadoss S, Lison D, Godderis L, et al. Toxicology of silica nanoparticles: an update. Arch Toxicol. 2017;91:2967. https:// doi.org/10.1007/s00204-017-1993-y.

Nagesetti A, McGoron A. Multifunctional organically modified silica nanoparticles for chemotherapy, adjuvant hyperthermia and near infrared imaging. Coll Surf B. 2016;147:482-500. https://doi.org/10.1016/j.colsurfb.2016.07.048.

Nehl C, Liao H, Hafne J. Optical properties of star-shaped gold nanoparticles. Nano Lett. 2006;6(4):683-8. https://doi. org/10.1021/nl052409y.

Nichols J, Bae Y. EPR: Evidence and fallacy. J Control Release. 2014;190:451-64. https://doi.org/10.1016/j.jconr el.2014.03.057.

Noel S, Liberelle B, Robitaille L, et al. Quantification of primary amine groups available for subsequent biofunctionalization of polymer surfaces. Bioconjug Chem. 2011;22(8):1690-9. https://doi.org/10.1021/bc200259c.

Nooney R, McCahey C, Stranik O, et al. Experimental and theoretical studies of optimisation of fluorescence from nearinfrared dye-doped silica nanoparticles. Anal Bioanal Chem. 2009;393:1143-9. https://doi.org/10.1007/s0021 6-008-2418-9.

Nooney R, McCormack E, McDonagh C. Optimization of size, morphology and colloidal stability of fluorescein dye-doped silica NPs for application in immunoassays. Anal Bioanal Chem. 2012;404(10):2807-18. https://doi.org/10.1007/ s00216-012-6224-z.

Nooney R, O'Connell C, Roy S, et al. Synthesis and characterisation of far-red fluorescent cyanine dye doped silica nanoparticles using a modified microemulsion method for application in bioassays. Sens Actuators B Chem. 2015;221:470-9. https://doi.org/10.1016/j.snb.2015.06.117.

O'Brown N, Pfau S, Gu C. Bridging barriers: a comparative look at the blood-brain barrier across organisms. Genes Dev. 2018;32:466-78. https://doi.org/10.1101/gad.309823.117.

O'Connell C, Nooney R, McDonagh C. Cyanine5-doped silica nanoparticles as ultra-bright immunospecific labels for model circulating tumour cells in flow cytometry and microscopy. Biosens Bioelectron. 2017;91:190-8. https:// doi.org/10.1016/j.bios.2016.12.023.

Ohulchanskyy T, Roy I, Goswami L, et al. Organically modified silica nanoparticles with covalently incorporated photosensitizer for photodynamic therapy of cancer. Nano Lett. 2007;7(9):2835-42. https://doi.org/10.1021/ nl0714637.

Oomen A, Steinhäuser K, Bleeker E, et al. Risk assessment frameworks for nanomaterials: scope, link to regulations, applicability, and outline for future directions in view of needed increase in efficiency. Nanolmpact. 2018;1:1-13.

Ow H, Larson D, Srivastava M, et al. Bright and stable core-shell fluorescent silica nanoparticles. Nano Lett. 2005;5(1):1137. https://doi.org/10.1021/nl0482478.

Paithankar D, Hwang B, Munavalli G, et al. Ultrasonic delivery of silica-gold nanoshells for photothermolysis of sebaceous glands in humans: nanotechnology from the bench to clinic. J Control Release. 2015;206:30-6. https://doi. org/10.1016/j.jconrel.2015.03.004.

Park J, von Maltzahn G, Zhang L, et al. Magnetic iron oxide nanoworms for tumor targeting and imaging. Adv Mater. 2008;20(9):1630-5. https://doi.org/10.1002/adma.200800004.

Park J, Gu L, von Maltzahn G, et al. Biodegradable luminescent porous silicon nanoparticles for in vivo applications. Nat Mater. 2009;8:331-6. https://doi.org/10.1038/nmat2398.

Paszek M, DuFort C, Rossier O, et al. The cancer glycocalyx mechanically primes integrin-mediated growth and survival. Nature. 2014;511(7509):319-25. https://doi.org/10.1038/nature13535.

Pedone A, Gambuzzi E, Barone V, et al. Understanding the photophysical properties of coumarin-based Pluronic-silica (PluS) nanoparticles by means of time-resolved emission spectroscopy and accurate TDDFT/stochastic calculations. Phys Chem Chem Phys. 2013;15(29):12360-72. https://doi.org/10.1039/c3cp51943k.

Peer D, Karp J, Hong S, et al. Nanocarriers as an emerging platform for cancer therapy. Nat Nanotechnol. 2007;2:751-60. https://doi.org/10.1038/nnano.2007.387.

Phillips E, Penate-Medina O, Zanzonico P, et al. Clinical translation of an ultrasmall inorganic optical-PET imaging nanoparticle probe. Sci Trans Med. 2014;6(260):260ra149. https://doi.org/10.1126/scitransImed.3009524.

Pisani C, Gaillard J, Dorandeu C, et al. Experimental separation steps influence the protein content of corona around mesoporous silica nanoparticles. Nanoscale. 2017;9:5769-72. https://doi.org/10.1039/c7nr01654a.

Poh T, Ali N, Mac Aogáin M, et al. Inhaled nanomaterials and the respiratory microbiome: clinical, immunological and toxicological perspectives. Part Fibre Toxicol. 2018;15(1):46. https://doi.org/10.1186/s12989-018-0282-0. 
Porchetta A, Vallee-Belisle A, Plaxco K, et al. Using distal site mutations and allosteric inhibition to tune, extend and narrow the useful dynamic range of aptamer-based sensors. J Am Chem Soc. 2012;134:51. https://doi.org/10.1021/ ja310585e.

Quan B, Choi K, Kim K, et al. Near infrared dye indocyanine green doped silica nanoparticles for biological imaging. Talanta. 2012;99:387-93. https://doi.org/10.1016/j.talanta.2012.05.069.

Rampazzo E, Boschi F, Bonacchi S, et al. Multicolor core/shell silica nanoparticles for in vivo and ex vivo imaging. Nanoscale. 2012;7(4):824-30. https://doi.org/10.1039/c1 nr11401h.

Ross J, Chaudhuri P, Ratnam M. Differential regulation of folate receptor isoforms in normal and malignant tissues in vivo and in established cell lines. Physiologic and clinical implications. Cancer. 1994;73(9):2432-43. https://doi. org/10.1002/1097-0142(19940501)73:9\%3c2432:aid-cncr2820730929\%3e3.0.co;2-s.

Rostovtsev V, Green L, Fokin V, et al. A stepwise Huisgen cycloaddition process: copper(I)-catalyzed regioselective "ligation" of azides and terminal alkynes. Angew Chem Int Ed. 2002;41(14):2596-9. https://doi.org/10.1002/15213773(20020715)41:14\%3c2596:aid-anie2596\%3e3.0.co;2-4.

Roy I, Kumar P, Kumar O, et al. Ormosil nanoparticles as a sustained-release drug delivery vehicle. RSC Adv. 2014;4:53498504. https://doi.org/10.1039/c4ra10293b.

Saha B, Evers T, Prins M. How antibody surface coverage on nanoparticles determines the activity and kinetics of antigen capturing for biosensing. Anal Chem. 2014;86(16):8158-66. https://doi.org/10.1021/ac501536z.

Salvati A, Pitek A, Monopoli M, et al. Transferrin-functionalized nanoparticles lose their targeting capabilities when a biomolecule corona adsorbs on the surface. Nat Nanotechnol. 2013;8:137-43. https://doi.org/10.1038/nnano .2012.237.

Sandoval K, Witt K. Blood-brain barrier tight junction permeability and ischemic stroke. Neurobiol Dis. 2008;32(2):200-19. https://doi.org/10.1016/j.nbd.2008.08.005.

Santra S, Yang H, Dutta D, et al. TAT conjugated, FITC doped silica nanoparticles for bioimaging applications. Chem Commun. 2004;24:2810-1. https://doi.org/10.1039/b411916a.

Santra S, Liesenfeld B, Dutta D, et al. Folate conjugated fluorescent silica nanoparticles for labeling neoplastic cells. J Nanosci Nanotechnol. 2005;5(6):899-904. https://doi.org/10.1166/jnn.2005.146.

Sapsford K, Algar W, Berti L, et al. Functionalizing nanoparticles with biological molecules: developing chemistries that facilitate nanotechnology. Chem Rev. 2013;113(3):1904-2074. https://doi.org/10.1021/cr300143v.

Sardesai N, Pana S, Rusling J. Electrochemiluminescent immunosensor for detection of protein cancer biomarkers using carbon nanotube forests and $\left[\mathrm{Ru}-(\mathrm{bpy})_{3}\right]^{2+}$-doped silica nanoparticles. Chem Commun. 2009. https://doi. org/10.1039/b909220j.

Schulz A, Woolley R, Tabarin T, et al. Dextran-coated silica nanoparticles for calcium-sensing. Analyst. 2011;136:1722. https ://doi.org/10.1039/c0an01009j.

Seferos D, Giljohann D, Hill H, et al. Nano-flares: probes for transfection and mRNA detection in living cells. J Am Chem Soc. 2007;129(50):15477-9. https://doi.org/10.1021/ja0776529.

Shahabi S, Treccani L, Dringen R, et al. Modulation of silica nanoparticle uptake into human osteoblast cells by variation of the ratio of amino and sulfonate surface groups: effects of serum. ACS Appl Mater Interfaces. 2015;7(25):13821-33. https://doi.org/10.1021/acsami.5b01900.

Shan G, Weissleder R, Hilderbrand S. Upconverting organic dye doped core-shell nano-composites for dual-modality NIR imaging and photo-thermal therapy. Theranostics. 2013;3(4):267-74. https://doi.org/10.7150/thno.5226.

Shang W, Nuffer J, Muñiz-Papandrea V, et al. Cytochrome c on silica nanoparticles: influence of nanoparticle size on protein structure, stability, and activity. Small. 2009;5(4):470-6. https://doi.org/10.1002/smll.200800995.

Sharma R, Das S, Maitra A. Surface modified ormosil nanoparticles. J Colloid Interface Sci. 2004;227(2):342-6. https:// doi.org/10.1016/j.jcis.2004.04.019.

Shen Z, Wu A, Chen W. Current detection technologies for circulating tumor cells. Chem Soc Rev. 2017;46:2038-56. https://doi.org/10.1016/j.cell.2017.01.026.

Sivaram A, Wardiana A, Howard C, et al. Recent advances in the generation of antibody-nanomaterial conjugates. Adv Healthc Mater. 2018. https://doi.org/10.1002/adhm.201700607.

Smith E, Chen W. How to prevent the loss of surface functionality derived from aminosilanes. Langmuir. 2008;24(21):12405-9. https://doi.org/10.1021/la802234x.

Soddu L, Trinh D, Dunne E, et al. Identification of the physical-chemical properties that modulate the nanoparticles aggregation in blood. Bellstein Arch. 2019. https://doi.org/10.3762/bxiv.2019.112.v1.

Song Y, Du D, Li L, et al. In vitro study of receptor-mediated silica nanoparticles delivery across blood-brain barrier. ACS Appl Mater Interfaces. 2017;9(24):20410-6. https://doi.org/10.1021/acsami.7b03504.

Stanimirovic D, Bani-Yaghoub M, Perkins M, et al. Blood-brain barrier models: in vitro to in vivo translation in preclinical development of CNS-targeting biotherapeutics. Expert Opin Drug Discov. 2014;10:1. https://doi. org/10.1517/17460441.2015.974545.

Stöber W, Fink A, Bohn E. Controlled growth of monodisperse silica spheres in the micron size range. J Colloid Interface Sci. 1968;26(1):62-9. https://doi.org/10.1016/0021-9797(68)90272-5.

Subiel A, Ashmore R, Schettino G. Standards and methodologies for characterizing radiobiological impact of high-Z nanoparticles. Theranostics. 2016;6(10):1651-71. https://doi.org/10.7150/thno.15019.

Sun $X$, Feng Z, Zhang $L$, et al. The selective interaction between silica nanoparticles and enzymes from molecular dynamics simulations. PLoS ONE. 2014;9(9):e107696. https://doi.org/10.1371/journal.pone.0107696.

Sun Q, Zhao G, Dou W. Blue silica nanoparticle-based colorimetric immunoassay for detection of Salmonella pullorum. Anal Methods. 2015;7:8647-54. https://doi.org/10.1039/c5ay02073e.

Sun Q, Zhao G, Dou W. An optical and rapid sandwich immunoassay method for detection of Salmonella pullorum and Salmonella gallinarum based on immune blue silica nanoparticles and magnetic nanoparticles. Sens Actuators B Chem. 2016;226:69-75. https://doi.org/10.1016/j.snb.2015.11.117.

Sun Y, Kunc F, Balhara V, et al. Quantification of amine functional groups on silica nanoparticles: a multi-method approach. Nanoscale Adv. 2019;1:1598-607. https://doi.org/10.1039/c9na00016j. 
Suzuki K, Sato S, Fujita M. Template synthesis of precisely monodisperse silica nanoparticles within self-assembled organometallic spheres. Nat Chem. 2010;2(1):25-9. https://doi.org/10.1038/nchem.446.

Sweeney M, Sagare A, Zlokovic B. Blood-brain barrier breakdown in Alzheimer disease and other neurodegenerative disorders. Nat Rev Neurol. 2018;14:133-50.

Tagaya M, Ikoma T, Yoshioka T, et al. Synthesis and luminescence properties of Eu(III)-doped nanoporous silica spheres. J Colloid Interface Sci. 2011;363(2):456-64. https://doi.org/10.1016/j.jcis.2011.07.066.

Tan Y, Liu M, Nolting B, et al. A nanoengineering approach for investigation and regulation of protein immobilzation. ACS Nano. 2008;2:2374-84. https://doi.org/10.1021/nn800508f.

Tenzer S, Docter D, Kuharev J, et al. Rapid formation of plasma protein corona critically affects nanoparticle pathophysiology. Nat Nanotechnol. 2013;8:772-81. https://doi.org/10.1038/nnano.2013.181.

Tighe P, Ryder R, Todd I, et al. ELISA in the multiplex era: potentials and pitfalls. Proteom Clin Appl. 2015;9:406-22. https://doi.org/10.1002/prca.201400130.

Tivnan A, Orr W, Gubala V, et al. Inhibition of neuroblastoma tumor growth by targeted delivery of MicroRNA-34a using anti-disialoganglioside GD2 coated nanoparticles. PLoS ONE. 2012;7(5):e38129. https://doi.org/10.1371/ journal.pone.0038129.

Toriyama K, Suzuki T, Inoue T, et al. Development of an immunochromatographic assay kit using fluorescent silica nanoparticles for rapid diagnosis of Acanthamoeba keratitis. J Clin Microbiol. 2015;53:273-7. https://doi. org/10.1128/jcm.02595-14.

Tornøe C, Christensen C, Meldal M. Peptidotriazoles on solid phase: [1,2,3]-triazoles by regiospecific copper(I)catalyzed 1,3-dipolar cycloadditions of terminal alkynes to azides. J Org Chem. 2002;67(9):3057-64. https://doi. org/10.1021/jo011148j.

Treerattrakoon K, Chanthima W, Apiwat C, et al. Oriented conjugation of antibodies against the epithelial cell adhesion molecule on fluorescently doped silica nanoparticles for flow-cytometric determination and in vivo imaging of EpCAM, a biomarker for colorectal cancer. Microchim Acta. 2017;184(7):1941-50. https://doi. org/10.1007/s00604-017-2211-6.

Truskey G. Human microphysiological systems and organoids as in vitro models for toxicological studies. Front Public Health. 2018;6:185. https://doi.org/10.3389/fpubh.2018.00185.

US FDA Center for Drug Evaluation and Research. Drug products, including biological products, that contain nanomaterials_ guidance for industry. 2017.

Van Blaaderen A, Vrij A. Synthesis and characterization of colloidal dispersions of fluorescent, monodisperse silica spheres. Langmuir. 1992;8(12):2921-31. https://doi.org/10.1021/la00048a013.

Vertegel A, Siegel R, Dordick J. Silica nanoparticle size influences the structure and enzymatic activity of adsorbed lysozyme. Langmuir. 2004;20:6800-7. https://doi.org/10.1021/la0497200.

Walczyk D, Bombelli F, Monopoli M, et al. What the cell "Sees" in bionanoscience. J Am Chem Soc. 2010;132:5761-8. https://doi.org/10.1021/ja910675v.

Wan S, Kelly P, Mahon E, et al. The "sweet" side of the protein corona: effects of glycosylation on nanoparticle-cell interactions. ACS Nano. 2015;9(2):2157-66. https://doi.org/10.1021/nn506060q.

Wang L, Tan W. Multicolor FRET silica nanoparticles by single wavelength excitation. Nano Lett. 2006;6(1):84-8. https ://doi.org/10.1021/nl052105b.

Wang L, Zhao W, Tan W. Bioconjugated silica nanoparticles: development and applications. Nano Res. 2008;1:99-115. https://doi.org/10.1007/s12274-008-8018-3.

Wang X, Yao S, Ahn H, et al. Folate receptor targeting silica nanoparticle probe for two-photon fluorescence bioimaging. Biomed Opt Express. 2010;1 (2):453-62. https://doi.org/10.1364/boe.1.000453.

Wang J, Sugawara-Narutaki A, Fukao M, et al. Two-phase synthesis of monodisperse silica nanospheres with amines or ammonia catalyst and their controlled self-assembly. ACS Appl Mater Interfaces. 2011a;3:1538-44. https:// doi.org/10.1021/am200104m.

Wang X, Morales A, Urakami T, et al. Folate receptor-targeted aggregation-enhanced near-ir emitting silica nanoprobe for one-photon in vivo and two-photon ex vivo fluorescence bioimaging. Bioconjug Chem. 2011b;22(7):1438-50. https://doi.org/10.1021/bc2002506.

Wang $K$, He X, Yang $X$, et al. Functionalized silica nanoparticles: a platform for fluorescence imaging at the cell and small animal levels. Acc Chem Res. 2013;46(7):1367-76. https://doi.org/10.1021/ar3001525.

Wang Z, Hong X, Zong S, et al. BODIPY-doped silica nanoparticles with reduced dye leakage and enhanced singlet oxygen generation. Sci Rep. 2015;5:12602. https://doi.org/10.1038/srep12602.

Wang Z, Wang C, Liu S, et al. Specifically formed corona on silica nanoparticles enhances transforming growth factor $\beta 1$ activity in triggering lung fibrosis. ACS Nano. 2017;28(11):1659-72. https://doi.org/10.1021/acsnano.6b074 61.

Wanga Y, Hu A. Carbon quantum dots: synthesis, properties and applications. J Mater Chem C. 2014;2:6921-39. https ://doi.org/10.1039/c4tc00988f.

Wegner D, Hildebrandt N. Quantum dots: bright and versatile in vitro and in vivo fluorescence imaging biosensors. Chem Soc Rev. 2015;44:4792. https://doi.org/10.1039/c4cs00532e.

Weitzmann M, Ha S, Vikulina T, et al. Bioactive silica nanoparticles reverse age-associated bone loss in mice. Nanomed Nanotechnol Biol Med. 2015;11:959-67. https://doi.org/10.1016/..nano.2015.01.013.

Wilhelm S, Tavares A, Dai Q, et al. Analysis of nanoparticle delivery to tumours. Nat Rev Mater. 2016;1:16104.

Wolfbeis O. An overview of nanoparticles commonly used in fluorescent bioimaging. Chem Soc Rev. 2015;44:4743. https://doi.org/10.1039/c4cs00392f.

Wu H, Huo Q, Varnum S, et al. Dye-doped silica nanoparticle labels/protein microarray for detection of protein biomarkers. Analyst. 2008a;133:1550-5. https://doi.org/10.1039/b719810h.

Wu P, He X, Wang K, et al. Imaging breast cancer cells and tissues using peptide-labeled fluorescent silica nanoparticles. J Nanosci Nanotechnol. 2008b;8(5):2483-7. https://doi.org/10.1166/jnn.2008.362.

Wu Y, Chen C, Liu S. Enzyme-functionalized silica nanoparticles as sensitive labels in biosensing. Anal Chem. 2009;81:1600-7. https://doi.org/10.1021/ac802345z. 
Wu Y, Wei P, Pengpumkiat S, et al. A novel ratiometric fluorescent immunoassay for human a-fetoprotein based on carbon nanodot-doped silica nanoparticles and FITC. Anal Methods. 2016;8:5398-406. https://doi. org/10.1039/c6ay01171c.

Wu L, Glebe U, Böker A. Fabrication of thermoresponsive plasmonic core-satellite nanoassemblies with a tunable stoichiometry via surface-initiated reversible addition-fragmentation chain transfer polymerization from silica nanoparticles. Adv Mater Interfaces. 2017:4(15):1700092. https://doi.org/10.1002/admi.201700092.

Xiao Y, Lai R, Plaxco K. Preparation of electrode-immobilized, redox-modified oligonucleotides for electrochemical DNA and aptamer-based sensing. Nat Protoc. 2007;2(11):2875-80. https://doi.org/10.1038/nprot.2007.413.

Yan J, Estevez M, Smith J, et al. Dye-doped nanoparticles for bioanalysis. Nanotoday. 2007;2:44-50. https://doi. org/10.1016/s1748-0132(07)70086-5.

Yang H, Lou C, Xu M, et al. Investigation of folate-conjugated fluorescent silica nanoparticles for targeting delivery to folate receptor-positive tumors and their internalization mechanism. Int J Nanomed. 2011;6(2023):2032. https ://doi.org/10.2147/ijn.s24792.

Yang Q, Jones S, Parker C, et al. Evading immune cell uptake and clearance requires PEG grafting at densities substantially exceeding the minimum for brush conformation. Mol Pharm. 2014;11(4):1250-8. https://doi. org/10.1021/mp400703d.

Yasun E, Li C, Barut l, et al. BSA modification to reduce CTAB induced nonspecificity and cytotoxicity of aptamerconjugated gold nanorods. Nanoscale. 2015;7:10240-8. https://doi.org/10.1039/c5nr01704a.

Ye S, Tian M, Wang T, et al. Synergistic effects of cell-penetrating peptide Tat and fusogenic peptide HA2-enhanced cellular internalization and gene transduction of organosilica nanoparticles. Nanomed Nanotechnol Biol Med. 2012;8:833-41. https://doi.org/10.1016/j.nano.2011.10.003.

Yokoi T, Wakabayashi J, Otsuka Y, et al. Mechanism of formation of uniform-sized silica nanospheres catalyzed by basic amino acids. Chem Mater. 2009;21(15):3719-29. https://doi.org/10.1021/cm900993b.

Yoo B, Ma K, Zhang L, et al. Ultrasmall dual-modality silica nanoparticle drug conjugates: design, synthesis, and characterization. Bioorg Med Chem. 2015;23:7119-30. https://doi.org/10.1016/j.bmc.2015.09.050.

Yu K, Grabinski C, Schrand A, et al. Toxicity of amorphous silica nanoparticles in mouse keratinocytes. J Nanopart Res. 2009;11:15-24. https://doi.org/10.1007/s11051-008-9417-9.

Zhang Q, Huang R, Guo L. One-step and high-density protein immobilization on epoxysilane-modified silica nanoparticles. Chin Sci Bull. 2009;54(15):2620-6. https://doi.org/10.1007/s11434-009-0210-7.

Zhang T, Li W, Meng G, et al. Strategies for transporting nanoparticles across the blood-brain barrier. Biomater Sci. 2016;4(2):219-29. https://doi.org/10.1039/c5bm00383k.

Zhao X, Wang J, Tao S, et al. In vivo bio-distribution and efficient tumor targeting of gelatin/silica nanoparticles for gene delivery. Nanoscale Res Lett. 2016;11:195. https://doi.org/10.1186/s11671-016-1409-6.

Zhu M, Lerum M, Chen W. How to prepare reproducible, homogeneous, and hydrolytically stable aminosilanederived layers on silica. Langmuir. 2012;28(1):416-23. https://doi.org/10.1021/la203638g.

Zielinskia J, Möller A, Frenz M, et al. Evaluation of endocytosis of silica particles used in biodegradable implants in the brain. Nanomed Nanotechnol Biol Med. 2016;12:1603-13. https://doi.org/10.1016/j.nano.2016.02.009.

Zou C, Foda M, Tan X, et al. Carbon-dot and quantum-dot-coated dual-emission core-satellite silica nanoparticles for ratiometric intracellular $\mathrm{Cu}^{2+}$ imaging. Anal Chem. 2016;88(14):7395-403. https://doi.org/10.1021/acs.analc hem.6b01941.

\section{Publisher's Note}

Springer Nature remains neutral with regard to jurisdictional claims in published maps and institutional affiliations.

Ready to submit your research? Choose BMC and benefit from:

- fast, convenient online submission

- thorough peer review by experienced researchers in your field

- rapid publication on acceptance

- support for research data, including large and complex data types

- gold Open Access which fosters wider collaboration and increased citations

- maximum visibility for your research: over $100 \mathrm{M}$ website views per year

At BMC, research is always in progress.

Learn more biomedcentral.com/submissions 